This is a self-archived version of an original article. This version may differ from the original in pagination and typographic details.

Author(s): Haslinger, Jaroslav; Sysala, Stanislav; Repin, Sergey

Title: Inf-sup conditions on convex cones and applications to limit load analysis

Year: 2019

Version: Accepted version (Final draft)

Copyright: (c) The Authors, 2019

Rights: In Copyright

Rights url: http://rightsstatements.org/page//nC/1.0/?language=en

Please cite the original version:

Haslinger, J., Sysala, S., \& Repin, S. (2019). Inf-sup conditions on convex cones and applications to limit load analysis. Mathematics and Mechanics of Solids, 24(10), 3331-3353.

https://doi.org/10.1177/1081286519843969 


\title{
Inf-sup conditions on convex cones and their applications to limit load analysis
}

\author{
J. Haslinger ${ }^{1,2}$, S. Repin ${ }^{3,4}$, S. Sysala ${ }^{1}$ \\ ${ }^{1}$ Institute of Geonics of the Czech Academy of Sciences, Ostrava, Czech Republic \\ ${ }^{2}$ Charles University in Prague, Prague, Czech Republic \\ ${ }^{3}$ St. Petersburg Department of V.A. Steklov Institute of Mathematics \\ of the Russian Academy of Sciences, Russia \\ ${ }^{4}$ University of Jyväskylä, Finland
}

November 6, 2018

\begin{abstract}
The paper is devoted to a family of specific inf-sup conditions on convex cones. We discuss their validity and consequences like estimates of the distance to these cones. Then, this theoretical background is applied to the limit analysis problem, which determines a safety parameter of a structure subject to external forces and a yield criterion. The equivalence between the static and kinematic approaches to limit analysis is proven and computable majorants of the limit load are derived. A particular interest is paied to the Drucker-Prager yield criterion and numerical examples including strip-footing or slope stability. Numerical results are verified by using the computable majorants and expected failure mechanisms are visualized.
\end{abstract}

Keywords: inf-sup conditions, convex cones, perfect plasticity, limit analysis, computable majorants, finite element method, geotechnical applications

Subject classification: 49M15, 74C05, 74S05, 90C25

\section{Introduction}

Usually, inf-sup conditions arise in analysis of saddle-point problems in order to guarantee their solvability. Practically all of them are related to cases, where the primal and dual variables (entering the inf-sup condition) are linear manifolds of certain Banach spaces. However, in some optimization problems and in non-linear problems of continuum mechanics, the variables may be constrained to belong to appropriate cones in Banach spaces [14]. Then, mathematical and numerical analysis requires studying inf-sup conditions on convex cones. To the best of our knowledge this question has not been yet deeply studied and the current paper is intended to partially fill up the gap.

In this paper, we are concerned with the validity, computable bounds and several applications of the following inf-sup condition:

$$
c_{*}:=\inf _{\substack{\tau L^{2}(\Omega ; \mathcal{C}) \\ \tau \neq 0}} \sup _{\substack{v \in \mathbb{V} \\ v \neq 0}} \frac{\int_{\Omega} \tau: \varepsilon(v) d x}{\|\tau\|_{\Omega}\|\nabla v\|_{\Omega}}>0,
$$


where

$$
\begin{aligned}
\mathbb{V} & :=\left\{v \in W^{1,2}\left(\Omega ; \mathbb{R}^{d}\right) \mid v=0 \text { on } \Gamma_{0}\right\}, \\
L^{2}(\Omega ; \mathcal{C}) & :=\left\{\tau \in L^{2}\left(\Omega ; \mathbb{M}_{\text {sym }}^{d \times d}\right) \mid \tau(x) \in \mathcal{C} \text { for a.a. } x \in \Omega\right\},
\end{aligned}
$$

$\Omega \subset \mathbb{R}^{d}, d=2,3$, is a bounded Lipschitz domain, $\Gamma_{0} \subset \partial \Omega$, meas $_{d-1} \Gamma_{0}>0, \mathcal{C}$ is a closed, convex cone with vertex at zero in the space $\mathbb{M}_{\text {sym }}^{d \times d}$ of symmetric $d \times d$ matrices with the biscalar product “:”, $\varepsilon(v):=\frac{1}{2}\left(\nabla v+(\nabla v)^{\top}\right)$, and $\|\cdot\|_{\Omega}$ stands for the $L^{2}$-norm of scalar, vector or matrix functions defined in $\Omega$. In continuum mechanics, the spaces $\mathbb{V}$ and $L^{2}\left(\Omega ; \mathbb{M}_{\text {sym }}^{d \times d}\right)$ consist of kinematic (velocity or displacement) and static (stress or strain) fields, respectively.

The inf-sup condition (1.1) covers several interesting cases depending on the choice of $\mathcal{C}$. For example, if $\mathcal{C}=\left\{\tau \in \mathbb{M}_{\text {sym }}^{d \times d} \mid \quad \tau=q I, \quad q \in \mathbb{R}\right\}$, where $I$ is the identity matrix, then $L^{2}(\Omega ; \mathcal{C})=$ $\left\{\tau \in L^{2}\left(\Omega ; \mathbb{M}_{\text {sym }}^{d \times d}\right) \mid \tau=q I, \quad q \in L^{2}(\Omega)\right\},\|\tau\|_{\Omega}=\sqrt{d}\left\|_{q}\right\|_{\Omega}$, and (1.1) becomes the well-known inf-sup condition for incompressible flow media:

$$
c_{\Omega}:=\inf _{\substack{q \in L^{2}(\Omega) \\ q \neq 0}} \sup _{\substack{v \in \mathbb{V} \\ v \neq 0}} \frac{\int_{\Omega} q \operatorname{div} v d x}{\|q\|_{\Omega}\|\nabla v\|_{\Omega}}=\sqrt{d} \inf _{\substack{\tau \in L^{2}(\Omega ; \mathcal{C}) \\ \tau \neq 0}} \sup _{\substack{v \in \mathbb{V} \\ v \neq 0}} \frac{\int_{\Omega} \tau: \varepsilon(v) d x}{\|\tau\|_{\Omega}\|\nabla v\|_{\Omega}}>0
$$

which is valid if $0<\operatorname{meas}_{d-1} \Gamma_{0}<\operatorname{meas}_{d-1} \partial \Omega$. For $\Gamma_{0}=\partial \Omega$ (i.e. $\mathbb{V}=W_{0}^{1,2}\left(\Omega ; \mathbb{R}^{d}\right)$ ), the infimum must be taken only over the subspace of $L^{2}(\Omega)$ being orthogonal to constant functions. Although this condition (or its equivalent forms that follow from the closed range theorem [3]) are known from seventies years (see $[2,1,5,18,20]$ ), analytical and semi-analytical bounds of $c_{\Omega}$ have been derived fairly recently (see, e.g., [22, 11, 27, 28, 29]).

In Section 2, we consider the condition (1.1) in abstract form and show that for some classes of cones it can be justified by means of (1.2). Moreover, we derive lower bounds of $c_{*}$ from the estimates of $c_{\Omega}$ and show that they may be computable.

In Section 3, we derive estimates of the distance between a function from $\mathbb{V}$ and the set

$$
\mathcal{K}=\left\{w \in \mathbb{V} \mid \varepsilon(w) \in \mathcal{C}^{-} \text {a.e. in } \Omega\right\},
$$

where $\mathcal{C}^{-}:=\left\{\eta \in \mathbb{M}_{\text {sym }}^{d \times d} \mid \tau: \eta \leq 0 \quad \forall \tau \in \mathcal{C}\right\}$ is the polar cone of $\mathcal{C}$, under the assumption (1.1). For this purpose, we represent $L^{2}(\Omega ; \mathcal{C})$ as the set of Lagrange multipliers to $\mathcal{K}$.

The rest of the paper is devoted to applications of the inf-sup condition (1.1) and the related distance estimate to stability problems solved by limit analysis. Our aim is to extend recent results from [31] to be applicable in a geotechnical practice.

Limit analysis is one of the main methods in geotechnical and other stability problems where a safety parameter of a structure is determined for a prescribed load and material parameters. The safety parameter is represented by the limit value of a load factor. Beyond the limit load, the body collapses. Unlike other methods, a classical theory of limit analysis enables us to define the limit load directly by a specific variational problem under the assumption that the material is perfectly plastic and obeys the associative flow rule. This problem can be formulated either in terms of stresses (the static approach) or in terms of velocities (the kinematic approach). Both of them are in mutual duality. For a recent literature survey, we refer to [32]. The mathematical background to the classical theory of limit analysis has been developped in $[36,12,30]$.

In Section 4, we introduce the limit analysis problem for an abstract set $B$ representing plastically admissible stress tensors. Further, we present a large class of the sets $B$ which are defined using the cone $\mathcal{C}$ and show that $\mathcal{K}$ is the constraint set in the kinematic setting of the respective limit analysis problem.

In Section 5, we demonstrate the importance of the distance estimate to $\mathcal{K}$ in limit analysis. Provided that (1.1) holds, we prove that the static and kinematic principles of limit analysis are equivalent and derive upper bounds of the limit load parameter using functions from a sufficiently 
large subset of $\mathbb{V}$. These bounds may be easily computable as soon as the lower bounds of $c_{*}$ are available.

In Section 6, we apply the results of Sections 2-5 to $B$ defined by the von Mises and DruckerPrager yield criteria, respectively. In the former case, we arrive at the known results from the recent paper [31]. As far as the latter case is concerned, we derive new results which can be useful in geotechnical practice.

In Section 7, we describe our computational strategy in kinematic limit analysis. Then, we present two numerical experiments with strip-footing and slope stability problems, where the DruckerPrager yield criterion will be used. Numerical results will be verified a posteriori by the computable majorants of the limit load.

\section{On validity of the inf-sup condition for abstract cone $\mathcal{C}$}

We start with a few general remarks on validity of the inf-sup condition (1.1). First, notice that the space $L^{2}\left(\Omega ; \mathbb{M}_{\text {sym }}^{d \times d}\right)$ of symmetric tensor fields can be additively decomposed into two closed and mutually orthogonal subspaces ([21]):

$$
\begin{aligned}
Q & :=\left\{\tau \in L^{2}\left(\Omega ; \mathbb{M}_{\text {sym }}^{d \times d}\right) \mid \int_{\Omega} \tau: \varepsilon(v) d x=0 \quad \forall v \in \mathbb{V}\right\}, \\
Q^{\perp} & :=\left\{\tau \in L^{2}\left(\Omega ; \mathbb{M}_{\text {sym }}^{d \times d}\right) \mid \exists v \in \mathbb{V}: \tau=\varepsilon(v)\right\}
\end{aligned}
$$

i.e., $L^{2}\left(\Omega ; \mathbb{M}_{\text {sym }}^{d \times d}\right)=Q \oplus Q^{\perp}$. It is easy to see that $Q$ contains tensor valued functions that satisfy (in a generalised sense) the equation $\operatorname{Div} \tau=0$ in $\Omega$ and the boundary condition $\tau \nu=0$ on $\partial \Omega \backslash \Gamma_{0}$, where $\nu$ denotes the unit outward normal to the boundary $\partial \Omega$. Moreover, there exists $\tau \in Q$ such that $\|\tau\|_{\Omega}>0$.

It is evident that the inf-sup condition (1.1) cannot hold if $L^{2}(\Omega ; \mathcal{C}) \cap Q \neq\{0\}$. For example, this occurs when $\mathcal{C}=\mathbb{M}_{\text {sym }}^{d \times d}$ or $\mathbb{V}=W_{0}^{1,2}\left(\Omega ; \mathbb{R}^{d}\right.$ ) (in the latter case, any constant tensor field belongs to $Q)$. Thus, here and in what follows, we shall suppose that

$$
0<\operatorname{meas}_{d-1} \Gamma_{0}<\operatorname{meas}_{d-1} \partial \Omega
$$

From the Korn inequality it follows the inf-sup condition

$$
\inf _{\substack{\tau \in Q^{\perp} \\ \tau \neq 0}} \sup _{\substack{v \in \mathbb{V} \\ v \neq 0}} \frac{\int_{\Omega} \tau: \varepsilon(v) d x}{\|\tau\|_{\Omega}\|\nabla v\|_{\Omega}}>0 .
$$

Unfortunately, the subspace $Q^{\perp}$ is too small to make conclusions for (1.1) to be satisfied. Therefore, to validate (1.1) we propose to use (1.2). Under the assumption (2.1), the inf-sup condition (1.2) is equivalent to the following statement: for any $q \in L^{2}(\Omega)$ there exists $v_{q} \in \mathbb{V}$ such that

$$
\operatorname{div} v_{q}=q, \quad\left\|\nabla v_{q}\right\|_{\Omega} \leq C_{\Omega}\|q\|_{\Omega}, \quad C_{\Omega}:=c_{\Omega}^{-1} .
$$

It is important to note that $C_{\Omega}^{2} \geq d^{-1}$ as follows from (1.2), the Hölder inequality, and $\|\operatorname{div} v\|_{\Omega} \leq$ $\sqrt{d}\|\nabla v\|_{\Omega}$. Next, we shall use the orthogonal decomposition of tensors:

$$
\tau=\frac{1}{d}(\operatorname{tr} \tau) I+\tau^{D}, \quad I: \tau^{D}=0, \quad|\tau|^{2}=\left|\tau^{D}\right|^{2}+d^{-1}(\operatorname{tr} \tau)^{2}, \quad \forall \tau \in \mathbb{M}_{\text {sym }}^{d \times d},
$$

where $I$ is the unit $d \times d$ matrix, $\operatorname{tr} \tau, \tau^{D}$ are the trace, and the deviatoric part of $\tau$, respectively, and $|\tau|^{2}:=\tau: \tau$. We have the following result. 
Theorem 2.1. Let $C_{\Omega}$ be defined by (2.2) and $\tilde{C}_{\Omega} \geq C_{\Omega}$. If $\mathcal{C} \in \mathbb{M}_{\text {sym }}^{d \times d}$ is a closed, convex cone with vertex at zero such that

$$
\exists \hat{a} \in \mathbb{R}, 0<\hat{a}<d^{-1}\left(\tilde{C}_{\Omega}^{2}-d^{-1}\right)^{-1 / 2}: \quad\left|\tau^{D}\right| \leq \hat{a}|\operatorname{tr} \tau| \quad \forall \tau \in \mathcal{C},
$$

then

$$
c_{*}:=\inf _{\substack{\tau \in L^{2}(\Omega ; \mathcal{C}) \\ \tau \neq 0}} \sup _{\substack{v \in \mathbb{V} \\ v \neq 0}} \frac{\int_{\Omega} \tau: \varepsilon(v) d x}{\|\tau\|_{\Omega}\|\nabla v\|_{\Omega}} \geq \frac{1-\hat{a} d \sqrt{\tilde{C}_{\Omega}^{2}-d^{-1}}}{\tilde{C}_{\Omega} \sqrt{\hat{a}^{2} d^{2}+d}}>0
$$

Proof. From (2.3) and (2.4), we have

$$
\left\|\tau^{D}\right\|_{\Omega} \leq \hat{a}\|\operatorname{tr} \tau\|_{\Omega}, \quad\|\tau\|_{\Omega}^{2}=\left\|\tau^{D}\right\|_{\Omega}^{2}+d^{-1}\|\operatorname{tr} \tau\|_{\Omega}^{2} \leq\left(\hat{a}^{2}+d^{-1}\right)\|\operatorname{tr} \tau\|_{\Omega}^{2} \quad \forall \tau \in L^{2}(\Omega ; \mathcal{C}) .
$$

Owing to $(2.2)$, for any $\tau \in L^{2}(\Omega ; \mathcal{C})$ there exists $v_{\tau} \in \mathbb{V}$ satisfying

$$
\operatorname{div} v_{\tau}=\operatorname{tr} \tau, \quad\left\|\nabla v_{\tau}\right\|_{\Omega} \leq \tilde{C}_{\Omega}\|\operatorname{tr} \tau\|_{\Omega}
$$

Since $\left\|\varepsilon\left(v_{\tau}\right)\right\|_{\Omega} \leq\left\|\nabla v_{\tau}\right\|_{\Omega}$, we have:

$$
\left\|\varepsilon^{D}\left(v_{\tau}\right)\right\|_{\Omega}^{2}=\left\|\varepsilon\left(v_{\tau}\right)\right\|_{\Omega}^{2}-d^{-1}\left\|\operatorname{div} v_{\tau}\right\|_{\Omega}^{2} \leq\left\|\nabla v_{\tau}\right\|_{\Omega}^{2}-d^{-1}\|\operatorname{tr} \tau\|_{\Omega}^{2} \stackrel{(2.7)}{\leq}\left(\tilde{C}_{\Omega}^{2}-d^{-1}\right)\|\operatorname{tr} \tau\|_{\Omega}^{2}
$$

Hence, for any $\tau \in L^{2}(\Omega ; \mathcal{C})$ :

$$
\begin{aligned}
& \sup _{\substack{v \in \mathbb{V} \\
v \neq 0}} \frac{\int_{\Omega} \tau: \varepsilon(v) d x}{\|\nabla v\|_{\Omega}} \geq \frac{\int_{\Omega} \tau: \varepsilon\left(v_{\tau}\right) d x}{\left\|\nabla v_{\tau}\right\|_{\Omega}}=\frac{\int_{\Omega}\left[\tau^{D}: \varepsilon^{D}\left(v_{\tau}\right)+d^{-1}(\operatorname{tr} \tau)\left(\operatorname{div} v_{\tau}\right)\right] d x}{\left\|\nabla v_{\tau}\right\|_{\Omega}} \\
& \stackrel{(2.7)}{\geq} \frac{-\left\|\tau^{D}\right\|_{\Omega}\left\|\varepsilon^{D}\left(v_{\tau}\right)\right\|_{\Omega}+d^{-1}\|\operatorname{tr} \tau\|_{\Omega}^{2}}{\tilde{C}_{\Omega}\|\operatorname{tr} \tau\|_{\Omega}} \\
& \stackrel{(2.6),(2.8)}{\geq} \frac{-\hat{a} \sqrt{\tilde{C}_{\Omega}^{2}-d^{-1}}\|\operatorname{tr} \tau\|_{\Omega}^{2}+d^{-1}\|\operatorname{tr} \tau\|_{\Omega}^{2}}{\tilde{C}_{\Omega}\|\operatorname{tr} \tau\|_{\Omega}} \\
& =\frac{1}{d \tilde{C}_{\Omega}}\left[1-\hat{a} d \sqrt{\tilde{C}_{\Omega}^{2}-d^{-1}}\right]\|\operatorname{tr} \tau\|_{\Omega} \\
& \stackrel{(2.6)}{\geq} \frac{1-\hat{a} d \sqrt{\tilde{C}_{\Omega}^{2}-d^{-1}}}{\tilde{C}_{\Omega} \sqrt{\hat{a}^{2} d^{2}+d}}\|\tau\|_{\Omega}
\end{aligned}
$$

Theorem 2.1 provides the lower bound (2.5) of the inf-sup constant $c_{*}$. To be this bound computable, we determine an appropriate estimate $\tilde{C}_{\Omega}$ of $C_{\Omega}$ which is based on the following inf-sup condition with the constant $C_{\Omega}^{0}[1,5,20]$ :

$$
\frac{1}{C_{\Omega}^{0}}:=\inf _{\substack{q \in L_{0}^{2}(\Omega) \\ q \neq 0}} \sup _{\substack{v \in W_{0}^{1,2}\left(\Omega ; \mathbb{R}^{d}\right) \\ v \neq 0}} \frac{\int_{\Omega} q \operatorname{div} v d x}{\|q\|_{\Omega}\|\nabla v\|_{\Omega}}>0
$$

where $L_{0}^{2}(\Omega):=\left\{q \in L^{2}(\Omega) \mid\{q\}_{\Omega}=0\right\}$ and $\{q\}_{\Omega}:=\frac{1}{|\Omega|} \int_{\Omega} q d x$. The following analogue of $(2.2)$ holds $[2,18,25,26]$ : for any $q \in L_{0}^{2}(\Omega)$ there exists $v_{q} \in W_{0}^{1,2}\left(\Omega ; \mathbb{R}^{d}\right)$ such that

$$
\operatorname{div} v_{q}=q, \quad\left\|\nabla v_{q}\right\|_{\Omega} \leq C_{\Omega}^{0}\|q\|_{\Omega}
$$


It is well-known that $C_{\Omega}^{0}$ depends only on $\Omega$. In addition, analytical bounds of $C_{\Omega}^{0}$ are available for special classes of domains $[22,11]$. For example, if $\Omega$ is a star-shaped $2 \mathrm{D}$ domain with respect to a ball of radius $\rho$ then

$$
C_{\Omega}^{0} \leq \tilde{C}_{\Omega}^{0}:=\frac{\sqrt{2}}{\kappa}\left(1+\sqrt{1-\kappa^{2}}\right)^{1 / 2}, \quad \kappa=\frac{\rho}{R},
$$

where $R$ is the radius of a concentric ball containing $\Omega$, see [11]. From this basic bound, one can derive semianalytical upper bounds of $C_{\Omega}^{0}$ and $C_{\Omega}$ by using weak solenoidal fields and domain decomposition techniques $[27,28,29,31]$. For purposes of numerical results presented in this paper, it will be sufficient to use (2.11) and the following result.

Lemma 2.1. Let (2.1) hold and $\tilde{C}_{\Omega}^{0}$ be an available upper bound of $C_{\Omega}^{0}$. Then

$$
C_{\Omega} \leq \tilde{C}_{\Omega}:=\sqrt{\left(\tilde{C}_{\Omega}^{0}\right)^{2}+|\Omega|^{-1}\|\nabla \tilde{v}\|_{\Omega}^{2}}, \quad \forall \tilde{v} \in \mathbb{V}, \operatorname{div} \tilde{v}=1 \text { in } \Omega .
$$

Proof. Let $q \in L^{2}(\Omega)$ be given and set $p:=q-\{q\}_{\Omega} \in L^{2}(\Omega),\{p\}_{\Omega}=0$. By (2.10), there exists $v_{p} \in W_{0}^{1,2}\left(\Omega ; \mathbb{R}^{d}\right)$ such that

$$
\operatorname{div} v_{p}=p, \quad\left\|\nabla v_{p}\right\|_{\Omega} \leq \tilde{C}_{\Omega}^{0}\|p\|_{\Omega}=\tilde{C}_{\Omega}^{0}\left\|q-\{q\}_{\Omega}\right\|_{\Omega} .
$$

Hence,

$$
\left\|\nabla v_{p}\right\|_{\Omega}^{2} \leq\left(\tilde{C}_{\Omega}^{0}\right)^{2}\|q\|_{\Omega}^{2}-\left(\tilde{C}_{\Omega}^{0}\right)^{2}|\Omega|\{q\}_{\Omega}^{2} .
$$

Further, there exists a function $\tilde{v} \in \mathbb{V}$ such that $\operatorname{div} \tilde{v}=1$ (see, e.g., [26, 35]). Then $v_{q}:=v_{p}+\{q\}_{\Omega} \tilde{v}$ satisfies

$$
\operatorname{div} v_{q}=\operatorname{div} v_{p}+\{q\}_{\Omega}=q
$$

and for any $\beta>0$,

$$
\begin{aligned}
\left\|\nabla v_{q}\right\|_{\Omega}^{2} & \leq(1+\beta)\left\|\nabla v_{p}\right\|_{\Omega}^{2}+\left(1+\beta^{-1}\right)\{q\}_{\Omega}^{2}\|\nabla \tilde{v}\|_{\Omega}^{2} \\
\stackrel{(2.14)}{\leq} & (1+\beta)\left(\tilde{C}_{\Omega}^{0}\right)^{2}\left(\|q\|_{\Omega}^{2}-|\Omega|\{q\}_{\Omega}^{2}\right)+\left(1+\beta^{-1}\right)\{q\}_{\Omega}^{2}\|\nabla \tilde{v}\|_{\Omega}^{2} \\
& =(1+\beta)\left(\tilde{C}_{\Omega}^{0}\right)^{2}\|q\|_{\Omega}^{2}+\left[\left(1+\beta^{-1}\right)\|\nabla \tilde{v}\|_{\Omega}^{2}-(1+\beta)\left(\tilde{C}_{\Omega}^{0}\right)^{2}|\Omega|\right]\{q\}_{\Omega}^{2} \\
& \leq(1+\beta)\left(\tilde{C}_{\Omega}^{0}\right)^{2}\|q\|_{\Omega}^{2}+\left[\left(1+\beta^{-1}\right)|\Omega|^{-1}\|\nabla \tilde{v}\|_{\Omega}^{2}-(1+\beta)\left(\tilde{C}_{\Omega}^{0}\right)^{2}\right]^{+}\|q\|_{\Omega}^{2} \\
& =\left((1+\beta)\left(\tilde{C}_{\Omega}^{0}\right)^{2}+\left[\left(1+\beta^{-1}\right)|\Omega|^{-1}\|\nabla \tilde{v}\|_{\Omega}^{2}-(1+\beta)\left(\tilde{C}_{\Omega}^{0}\right)^{2}\right]^{+}\right)\|q\|_{\Omega}^{2},
\end{aligned}
$$

where $s^{+}:=\max \{0, s\}$. To optimize this bound we set $\beta=\left(\tilde{C}_{\Omega}^{0}\right)^{-2}|\Omega|^{-1}\|\nabla \tilde{v}\|_{\Omega}^{2}$ leading to $\left\|\nabla v_{q}\right\|_{\Omega} \leq$ $\tilde{C}_{\Omega}\|q\|_{\Omega}$ for any $q \in L^{2}(\Omega)$. From this we arrive at (2.12).

\section{The inf-sup condition and related distance estimate}

In this section, we shall assume that (1.1) holds and derive a uniform distance estimate between a function from $\mathbb{V}$ and the set $\mathcal{K}$ defined by (1.3).

First, we summarize basic properties of the cones $\mathcal{C}$ and $\mathcal{C}^{-}=\left\{\eta \in \mathbb{M}_{\text {sym }}^{d \times d} \mid \tau: \eta \leq 0 \quad \forall \tau \in \mathcal{C}\right\}$. These cones have the vertex at zero and are closed and convex. Further, it is well-known that there exists a unique additive decomposition of any element $e \in \mathbb{M}_{\text {sym }}^{d \times d}: e=\tau_{e}+\eta_{e}$ such that $\tau_{e} \in \mathcal{C}$, $\eta_{e} \in \mathcal{C}^{-}$and $\tau_{e}: \eta_{e}=0$. In particular, it holds that

$$
\left|e-\tau_{e}\right| \leq|e-\tau| \quad \forall \tau \in \mathcal{C}, \text { i.e., } \tau_{e}=\Pi_{\mathcal{C}} e,
$$


where $\Pi_{\mathcal{C}}$ is the projection of $\mathbb{M}_{\text {sym }}^{d \times d}$ onto $\mathcal{C}$. Using the cone property of $\mathcal{C}$, problem (3.1) is equivalent to

$$
\left(e-\Pi_{\mathcal{C}} e\right): \Pi_{\mathcal{C}} e=0, \quad\left(e-\Pi_{\mathcal{C}} e\right): \tau \leq 0, \quad \forall \tau \in \mathcal{C} .
$$

From (3.1) and (3.2) it is easily seen that

$$
\begin{gathered}
\Pi_{\mathcal{C}}(\alpha e)=\alpha \Pi_{\mathcal{C}} e \quad \forall \alpha \geq 0, \forall e \in \mathbb{M}_{\text {sym }}^{d \times d}, \\
\left|\Pi_{\mathcal{C}} e\right|^{2}=\max _{\tau \in \mathcal{C}}\left\{-|\tau|^{2}+2 \tau: e\right\} \quad \forall e \in \mathbb{M}_{\text {sym }}^{d \times d} .
\end{gathered}
$$

To simplify notation, we use the same symbol $\Pi_{\mathcal{C}}$ to denote the projection of $L^{2}\left(\Omega ; \mathbb{M}_{\text {sym }}^{d \times d}\right)$ onto $L^{2}(\Omega ; \mathcal{C})$. Its definition and properties follow directly from $(3.2)-(3.4)$. Next, we derive the following distance estimate.

Theorem 3.1. Let the inf-sup condition (1.1) hold with the constant $c_{*}>0$. Then

$$
\min _{w \in \mathcal{K}}\|\nabla(v-w)\|_{\Omega} \leq \frac{1}{c_{*}}\left\|\Pi_{\mathcal{C}} \varepsilon(v)\right\|_{\Omega} \quad \forall v \in \mathbb{V} .
$$

Proof. The proof is inspired by $\left[25\right.$, Section 5.1]. Let $v \in \mathbb{V}$ be fixed and denote $\mathcal{J}_{v}(w)=\|\nabla(v-w)\|_{\Omega}^{2}$, $w \in \mathbb{V}$. Since $\mathcal{K} \neq \emptyset(0 \in \mathcal{K})$, there exists a unique minimizer $w^{*} \in \mathcal{K}$ of $\mathcal{J}_{v}$ on $\mathcal{K}$. To release the constraint $\varepsilon(w) \in \mathcal{C}^{-}$a.e. in $\Omega$ appearing in (1.3), we introduce the Lagrangian $\mathcal{L}_{v}: \mathbb{V} \times L^{2}(\Omega ; \mathcal{C}) \rightarrow$ $\mathbb{R}$ :

$$
\mathcal{L}_{v}(w, \tau)=\|\nabla(v-w)\|_{\Omega}^{2}+2 \int_{\Omega} \tau: \varepsilon(w) d x=\|\nabla(v-w)\|_{\Omega}^{2}+2 \int_{\Omega} \tau: \nabla w d x .
$$

Then from the definition of $\mathcal{C}^{-}$, we have:

$$
\min _{w \in \mathcal{K}}\|\nabla(v-w)\|_{\Omega}^{2}=\min _{w \in \mathbb{V}} \sup _{\tau \in L^{2}(\Omega ; \mathcal{C})} \mathcal{L}_{v}(w, \tau)
$$

To show that there exists a saddle-point of $\mathcal{L}_{v}$ in $\mathbb{V} \times L^{2}(\Omega ; \mathcal{C})$, we analyze the related dual problem:

$$
\sup _{\tau \in L^{2}(\Omega ; \mathcal{C})} \mathcal{S}_{v}(\tau), \quad \mathcal{S}_{v}(\tau):=\inf _{w \in \mathbb{V}} \mathcal{L}_{v}(w, \tau) .
$$

Using the substitution $w:=v+w$, the functional $\mathcal{S}_{v}$ can be written as

$$
\mathcal{S}_{v}(\tau)=\inf _{w \in \mathbb{V}}\left[\|\nabla w\|_{\Omega}^{2}+2 \int_{\Omega} \tau: \nabla w d x\right]+2 \int_{\Omega} \tau: \nabla v d x
$$

Notice that for any $\tau \in L^{2}(\Omega ; \mathcal{C})$ there exists a unique minimizer $w_{\tau} \in \mathbb{V}$ of the functional in the square brackets. It satisfies the equation

$$
\int_{\Omega} \nabla w_{\tau}: \nabla w d x=-\int_{\Omega} \tau: \nabla w d x \quad \forall w \in \mathbb{V}
$$

which implies

$$
\left\|\nabla w_{\tau}\right\|_{\Omega}=\sup _{\substack{w \in \mathbb{V} \\ w \neq 0}} \frac{\int_{\Omega} \tau: \nabla w d x}{\|\nabla w\|_{\Omega}}=:\|\tau\|_{*}
$$

Hence

$$
\inf _{w \in \mathbb{V}}\left[\|\nabla w\|_{\Omega}^{2}+2 \int_{\Omega} \tau: \nabla w d x\right]=-\left\|\nabla w_{\tau}\right\|_{\Omega}^{2}=-\left(\sup _{\substack{w \in \mathbb{V} \\ w \neq 0}} \frac{\int_{\Omega} \tau: \nabla w d x}{\|\nabla w\|_{\Omega}}\right)^{2}
$$


so that

$$
-\mathcal{S}_{v}(\tau)=\left(\sup _{\substack{w \in \mathbb{V} \\ w \neq 0}} \frac{\int_{\Omega} \tau: \nabla w d x}{\|\nabla w\|_{\Omega}}\right)^{2}-2 \int_{\Omega} \tau: \nabla v d x .
$$

From the assumption (1.1), we get

$$
\sup _{\substack{w \in \mathbb{V} \\ w \neq 0}} \frac{\int_{\Omega} \tau: \nabla w d x}{\|\nabla w\|_{\Omega}} \geq c_{*}\|\tau\|_{\Omega} \quad \forall \tau \in L^{2}(\Omega ; \mathcal{C}) .
$$

Therefore, $-\mathcal{S}_{v}$ is coercive in $L^{2}(\Omega ; \mathcal{C})$. Since $-\mathcal{S}_{v}$ is also convex and weakly lower semicontinuous in $L^{2}(\Omega ; \mathcal{C})$, there exists $\tau^{*} \in L^{2}(\Omega ; \mathcal{C})$ such that

$$
\mathcal{S}_{v}\left(\tau^{*}\right)=\max _{\tau \in L^{2}(\Omega ; \mathcal{C})} \mathcal{S}_{v}(\tau)
$$

By [14, Proposition VI.2.4], the pair $\left(w^{*}, \tau^{*}\right)$ is a saddle point of $\mathcal{L}_{v}$ in $\mathbb{V} \times L^{2}(\Omega ; \mathcal{C})$ and

$$
\min _{w \in \mathcal{K}}\|\nabla(v-w)\|_{\Omega}^{2}=\mathcal{J}_{v}\left(w^{*}\right)=\mathcal{L}_{v}\left(w^{*}, \tau^{*}\right)=\mathcal{S}_{v}\left(\tau^{*}\right)=\max _{\tau \in L^{2}(\Omega ; \mathcal{C})} \mathcal{S}_{v}(\tau)
$$

From this and (3.11), we easily obtain the required distance estimate. Indeed,

$$
\begin{aligned}
\min _{w \in \mathcal{K}}\|\nabla(v-w)\|_{\Omega}^{2} & =\max _{\tau \in L^{2}(\Omega ; \mathcal{C})} \mathcal{S}_{v}(\tau) \leq-\min _{\tau \in L^{2}(\Omega ; \mathcal{C})}\left\{c_{*}^{2}\|\tau\|_{\Omega}^{2}-2 \int_{\Omega} \tau: \nabla v d x\right\} \\
& =c_{*}^{2} \max _{\tau \in L^{2}(\Omega ; \mathcal{C})}\left\{-\|\tau\|_{\Omega}^{2}+2 \int_{\Omega} \tau: \frac{\varepsilon(v)}{c_{*}^{2}} d x\right\} \\
& \stackrel{(3.4)}{=} c_{*}^{2}\left\|\Pi_{\mathcal{C}}\left[\varepsilon(v) / c_{*}^{2}\right]\right\|_{\Omega}^{2} \stackrel{(3.3)}{=} \frac{1}{c_{*}^{2}}\left\|\Pi_{\mathcal{C}} \varepsilon(v)\right\|_{\Omega}^{2} \quad \forall v \in \mathbb{V} .
\end{aligned}
$$

Remark 3.1. Clearly, one can replace the constant $c_{*}^{-1}$ in the distance estimate (3.5) by its upper bound $\tilde{C}_{*} \geq c_{*}^{-1}$. If the assumptions of Theorem 2.1 are satisfied, one can set

$$
\tilde{C}_{*}=\frac{\tilde{C}_{\Omega} \sqrt{\hat{a}^{2} d^{2}+d}}{1-\hat{a} d \sqrt{\tilde{C}_{\Omega}^{2}-d^{-1}}}
$$

to be the distance estimate computable.

Remark 3.2. As mentioned in Section 2, the inf-sup condition (1.1) does not hold if there exists $\bar{\tau} \in L^{2}(\Omega, \mathcal{C}) \cap Q, \bar{\tau} \neq 0$, i.e.,

$$
\int_{\Omega} \bar{\tau}: \varepsilon(v) d x=0 \quad \forall v \in \mathbb{V} .
$$

In this case, it is readily seen that $\bar{\tau}: \varepsilon(w)=0$ a.e. in $\Omega$ for any $w \in \mathcal{K}$ which means that

$$
\begin{aligned}
\mathcal{K} & =\left\{w \in \mathbb{V} \mid \varepsilon(w) \in \mathcal{C}^{-} \text {a.e. in } \Omega\right\} \\
& =\left\{w \in \mathbb{V} \mid \varepsilon(w) \in \mathcal{C}^{-}, \bar{\tau}: \varepsilon(w)=0 \text { a.e. in } \Omega\right\}
\end{aligned}
$$

This additional constraint significantly influences the definition of $\mathcal{K}$. In particular, if $\Gamma_{0}=\partial \Omega$ then all constant tensor fields belong to $L^{2}(\Omega, \mathcal{C}) \cap Q$ and the additional constraints cause that $\mathcal{K}$ is even a linear subspace of $\mathbb{V}$. Moreover, if the interior of $\mathcal{C}$ is nonempty and $\Gamma_{0}=\partial \Omega$ then $\mathcal{K}=\{0\}$. 


\section{Limit analysis problem and its relationship to the set $\mathcal{K}$}

In this section, we introduce the static and kinematic formulations of the limit analysis problem and relate them to the cone $\mathcal{C}$ and the constraint set $\mathcal{K}$ introduced in the previous sections.

We consider that the body occupies the domain $\Omega$ and is fixed along $\Gamma_{0} \subset \partial \Omega$. On $\Gamma_{1}:=\partial \Omega \backslash \bar{\Gamma}_{0}$, surface forces of density $f \in L^{2}\left(\Gamma_{1} ; \mathbb{R}^{d}\right)$ are applied. Finally, $\Omega$ is subject to volume forces of density $F \in L^{2}\left(\Omega ; \mathbb{R}^{d}\right)$. The corresponding load functional reads as

$$
L(v):=\int_{\Omega} F \cdot v d x+\int_{\Gamma_{f}} f \cdot v d s, \quad v \in \mathbb{V} .
$$

The space $\mathbb{M}_{s y m}^{d \times d}$ is used for Cauchy stress and infinitisimal small strain tensors. The following two sets define statically and plastically admissible stress fields, respectively:

$$
\begin{aligned}
Q_{\lambda} & :=\left\{\tau \in L^{2}\left(\Omega, \mathbb{M}_{\text {sym }}^{d \times d}\right) \mid \int_{\Omega} \tau: \varepsilon(v) d x=\lambda L(v) \quad \forall v \in \mathbb{V}\right\}, \\
P & :=\left\{\tau \in L^{2}\left(\Omega, \mathbb{M}_{\text {sym }}^{d \times d}\right) \mid \tau(x) \in B \text { for a.a. } x \in \Omega\right\},
\end{aligned}
$$

where $\lambda \geq 0$ is the load parameter and

$$
B \subset \mathbb{M}_{\text {sym }}^{d \times d} \text { is closed, convex and } 0 \in \operatorname{int} B .
$$

The set $B$ is usually defined by a yield criterion, see Sections 6.1 and 6.2. For the sake of simplicity, we consider only homogeneous media and assume that $B$ is independent of the space variable $x \in \Omega$. Further, any $\tau \in Q_{\lambda}$ satisfies (in a generalized sense) the balance equation $\operatorname{Div} \tau+\lambda F$ in $\Omega$ and the Neumann boundary condition $\tau \nu=\lambda f$ on $\Gamma_{1}$. Clearly, if $\lambda=0$ then $Q_{\lambda}=Q$.

The static setting of the limit analysis problem defines the limit (ultimate) value $\lambda^{*}$ of the load parameter as follows:

$$
\lambda^{*}:=\sup \left\{\lambda \geq 0 \mid Q_{\lambda} \cap P \neq \emptyset\right\} .
$$

We see that there is no admissible stress state for loads $\lambda L, \lambda>\lambda^{*}$, meaning that the body collapses beyond the limit load. So finding $\lambda^{*}$ or some realistic bounds of this quantity is of practical interest.

The kinematic approach to limit analysis is based on the minimization of the plastic dissipation functional subject to the load constraint:

$$
(\mathcal{P})^{\infty} \quad \zeta^{*}:=\inf _{\substack{v \in \mathbb{V} \\ L(v)=1}} J_{\infty}(v), \quad J_{\infty}(v):=\int_{\Omega} j_{\infty}(\varepsilon(v)) d x, \quad v \in \mathbb{V},
$$

where

$$
j_{\infty}: \mathbb{M}_{s y m}^{d \times d} \rightarrow \overline{\mathbb{R}}_{+}, \quad \overline{\mathbb{R}}_{+}:=\mathbb{R}_{+} \cup\{+\infty\}, \quad j_{\infty}(e):=\sup _{\tau \in B} \tau: e, \quad e \in \mathbb{M}_{s y m}^{d \times d} .
$$

The function $j_{\infty}$ need not be finite everywhere and thus additional constraints in $(\mathcal{P})^{\infty}$ depending on the definition of $B$ may appear. Further, it is worth noticing that the space $\mathbb{V}$ is sufficient for the definition of the kinematic limit value $\zeta^{*}$ but the minimum of $J_{\infty}$ need not belong to $\mathbb{V}$, in general. To find a minimizer in (4.4), it is necessary to extend the space $\mathbb{V}$ and consider a relaxed problem, see $[36,12,30]$. The minimizers may be discontinuous along some $(d-1)$-dimensional zones characterizing a failure caused by the limit load.

The following duality relationship between the static and kinematic approaches holds [36]:

$$
\lambda^{*}=\sup _{\tau \in P} \inf _{\substack{v \in \mathbb{V} \\ L(v)=1}} \int_{\Omega} \tau: \varepsilon(v) \mathrm{d} x \leq \inf _{\substack{v \in \mathbb{V} \\ L(v)=1}} \int_{\Omega} j_{\infty}(\varepsilon(v)) \mathrm{d} x=\zeta^{*} .
$$




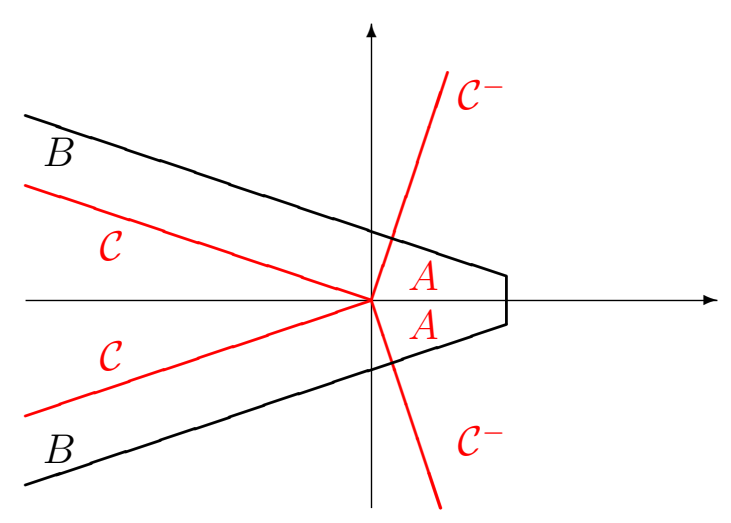

Figure 1: Scheme of the additive splitting of $B$.

We see that the kinematic limit value is only an upper bound of the "safety" value $\lambda^{*}$. However, the equality $\lambda^{*}=\zeta^{*}$ has been established for some yield criteria [36, 30,31]. Our aim is to prove this equality and derive a computable majorant of $\zeta^{*}$ within the framework of the results presented in previous sections.

To this end we shall consider the following additional assumption on the set $B$ :

$$
B=\mathcal{C}+A, \quad A:=B \cap \mathcal{C}^{-},
$$

where $\mathcal{C} \subset \mathbb{M}_{\text {sym }}^{d \times d}$ is a closed, convex cone with vertex at zero, $\mathcal{C}^{-}$is the polar cone to $\mathcal{C}$ and $A$ is bounded. From (4.2) and (4.6) it follows that $\mathcal{C}$ is the largest cone with vertex at zero which is contained in $B$ and $A$ is closed and convex, see Figure 1. We see that $B$ has a conical (or cylindrical) shape in the part $B \backslash \mathcal{C}^{-}$, while the shape of $B$ may be more general in $A=B \cap \mathcal{C}^{-}$. Let us note that (4.6) holds for sets $B$ which represent the classical yield criteria (i.e., the von Mises, Tresca, Drucker-Prager and Mohr-Coulomb ones [13]). The von Mises and Drucker-Prager yield criteria will be presented in Section 6.

Lemma 4.1. Let $B$ satisfy (4.2) and (4.6) with the cones $\mathcal{C}, \mathcal{C}^{-}$and $A=B \cap \mathcal{C}^{-}$. Then

$$
j_{\infty}(e)=\sup _{\tau \in B} \tau: e=\left\{\begin{array}{cc}
j_{\infty}^{A}(e), & e \in \mathcal{C}^{-}, \\
+\infty, & e \notin \mathcal{C}^{-},
\end{array} \quad j_{\infty}^{A}(e):=\max _{\tau \in A} \tau: e, \quad \forall e \in \mathbb{M}_{\text {sym }}^{d \times d},\right.
$$

and $\operatorname{dom} j_{\infty}=\mathcal{C}^{-}$.

Proof. Since $A$ is closed and bounded, the function $j_{\infty}^{A}$ is finite-valued in $\mathbb{M}_{s y m}^{d \times d}$. Further,

$$
\sup _{\tau \in B} \tau: e \stackrel{(4.6)}{=} \sup _{\tau_{\mathcal{C}} \in \mathcal{C}} \sup _{\tau_{A} \in A}\left(\tau_{\mathcal{C}}+\tau_{A}\right): e=\sup _{\tau \in \mathcal{C}} \tau: e+j_{\infty}^{A}(e) \quad \forall e \in \mathbb{M}_{\text {sym }}^{d \times d}
$$

To complete the proof, we show that $\psi(e):=\sup _{\tau \in \mathcal{C}} \tau: e$ is the indicator function to $\mathcal{C}^{-}$. Indeed, if $e \in \mathcal{C}^{-}$then $\psi(e)=0$, otherwise, $\Pi_{\mathcal{C}} e \neq 0$. Hence

$$
\begin{gathered}
\psi(e)=\sup _{\tau \in \mathcal{C}}\left\{\tau: \Pi_{\mathcal{C}} e+\tau:\left(e-\Pi_{\mathcal{C}} e\right)\right\} \geq \sup _{k \geq 0}\left\{k\left|\Pi_{\mathcal{C}} e\right|^{2}+k \Pi_{\mathcal{C}} e:\left(e-\Pi_{\mathcal{C}} e\right)\right\} \\
\stackrel{(3.2)}{=}\left|\Pi_{\mathcal{C}} e\right|^{2} \sup _{k \geq 0}\{k\}=+\infty \quad \forall e \notin \mathcal{C} .
\end{gathered}
$$


Notice that the function $j_{\infty}^{A}$ introduced in (4.7) satisfies

$$
\begin{gathered}
j_{\infty}^{A}(e) \leq \rho_{A}|e| \quad \forall e \in \mathbb{M}_{\text {sym }}^{d \times d}, \quad \rho_{A}:=\max _{\tau \in A}|\tau|, \\
j_{\infty}^{A}(e)-j_{\infty}^{A}(\bar{e}) \leq j_{\infty}^{A}(e-\bar{e}) \quad \forall e, \bar{e} \in \mathbb{M}_{\text {sym }}^{d \times d} .
\end{gathered}
$$

Further, from Lemma 4.1 it follows that the problem (4.4) can be written as

$$
\zeta^{*}=\inf _{\substack{v \in \mathcal{K} \\ L(v)=1}} J_{\infty}^{A}(v), \quad J_{\infty}^{A}(v):=\int_{\Omega} j_{\infty}^{A}(\varepsilon(v)) d x,
$$

where $\mathcal{K}$ is defined by (1.3). Formulation (4.10) is more convenient and transparent since the constraint set is specified and the functional $J_{\infty}^{A}$ is finite-valued in $\mathbb{V}$ as follows from (4.8).

\section{Applications of the distance estimate to limit analysis}

In this section, we derive a computable majorant of $\zeta^{*}$ and prove $\lambda^{*}=\zeta^{*}$ provided that the assumptions (1.1), (4.2) and (4.6) are satisfied.

\subsection{The upper bound of $\zeta^{*}$}

For any $w \in \mathcal{K}$ such that $L(w)>0$ (provided that such $w$ exists), we obtain the simple upper bound

$$
\zeta^{*} \leq \frac{J_{\infty}^{A}(w)}{L(w)},
$$

which follows from (4.10) using that $j_{\infty}^{A}$ is 1-positively homogeneous. We aim to derive upper bounds of $\zeta^{*}$ by means of functions which need not belong to $\mathcal{K}$.

Theorem 5.1. Let (1.1) be satisfied with $c_{*}>0$, (4.2), (4.6) hold, and

$$
\|L\|_{*}:=\sup _{\substack{v \in \mathbb{V} \\ v \neq 0}} \frac{L(v)}{\|\nabla v\|_{\Omega}} .
$$

Let $\tilde{C}_{*} \geq c_{*}^{-1},\|L\|_{+} \geq\|L\|_{*}$, and $\rho_{A}>0$ be defined by (4.8). Then for any $v \in \mathbb{V}$ such that $L(v)>\tilde{C}_{*}\|L\|_{+}\left\|\Pi_{\mathcal{C}} \varepsilon(v)\right\|_{\Omega}$, it holds:

$$
\zeta^{*} \leq \frac{J_{\infty}^{A}(v)+\tilde{C}_{*} \rho_{A}|\Omega|^{1 / 2}\left\|\Pi_{\mathcal{C}} \varepsilon(v)\right\|_{\Omega}}{L(v)-\tilde{C}_{*}\|L\|_{+}\left\|\Pi_{\mathcal{C}} \varepsilon(v)\right\|_{\Omega}}
$$

Proof. Owing to (1.1), the distance estimate (3.5) holds, i.e., for any $v \in \mathbb{V}$ there exists $w_{v} \in \mathcal{K}$ such that

$$
\left\|\nabla\left(v-w_{v}\right)\right\|_{\Omega} \leq \tilde{C}_{*}\left\|\Pi_{\mathcal{C}} \varepsilon(v)\right\|_{\Omega} .
$$

Let $v \in \mathbb{V}$ be given and $w_{v} \in \mathcal{K}$ satisfies (5.4). Then for any $\lambda \geq 0$ we have:

$$
\begin{aligned}
J_{\infty}^{A}\left(w_{v}\right)-\lambda L\left(w_{v}\right) & =J_{\infty}^{A}(v)-\lambda L(v)+\int_{\Omega}\left(j_{\infty}^{A}\left(\varepsilon\left(w_{v}\right)\right)-j_{\infty}^{A}(\varepsilon(v))\right) d x-\lambda L\left(w_{v}-v\right) \\
& \stackrel{(4.9)}{\leq} J_{\infty}^{A}(v)-\lambda L(v)+\int_{\Omega} j_{\infty}^{A}\left(\varepsilon\left(w_{v}-v\right)\right) d x+\lambda\|L\|_{+}\left\|\nabla\left(v-w_{v}\right)\right\|_{\Omega} \\
& \stackrel{(4.8)}{\leq} J_{\infty}^{A}(v)-\lambda L(v)+\left(\rho_{A}|\Omega|^{1 / 2}+\lambda\|L\|_{+}\right)\left\|\nabla\left(v-w_{v}\right)\right\|_{\Omega} \\
& \stackrel{(5.4)}{\leq} J_{\infty}^{A}(v)-\lambda L(v)+\tilde{C}_{*}\left(\rho_{A}|\Omega|^{1 / 2}+\lambda\|L\|_{+}\right)\left\|\Pi_{\mathcal{C}} \varepsilon(v)\right\|_{\Omega} \\
& =J_{\infty}^{A}(v)+\tilde{C}_{*} \rho_{A}|\Omega|^{1 / 2}\left\|\Pi_{\mathcal{C}} \varepsilon(v)\right\|_{\Omega}-\lambda\left(L(v)-\tilde{C}_{*}\|L\|_{+}\left\|\Pi_{\mathcal{C}} \varepsilon(v)\right\|_{\Omega}\right) .
\end{aligned}
$$


In addition, if $v$ is such that $L(v)>\tilde{C}_{*}\|L\|_{+}\left\|\Pi_{\mathcal{C}} \varepsilon(v)\right\|_{\Omega}$, then

$$
J_{\infty}^{A}\left(w_{v}\right)-\lambda L\left(w_{v}\right)<0 \quad \text { for any } \lambda>\frac{J_{\infty}^{A}(v)+\tilde{C}_{*} \rho_{A}|\Omega|^{1 / 2}\left\|\Pi_{\mathcal{C}} \varepsilon(v)\right\|_{\Omega}}{L(v)-\tilde{C}_{*}\|L\|_{+}\left\|\Pi_{\mathcal{C}} \varepsilon(v)\right\|_{\Omega}} .
$$

Since $w_{v} \in \mathcal{K}$, we arrive at (5.3) making use of (5.1).

One can see that the bounds (5.1) and (5.3) coincide for $v \in \mathcal{K}, L(v)>0$ as $\Pi_{\mathcal{C}} \varepsilon(v)=0$ for any $v \in \mathcal{K}$. Further, (5.3) is fully computable and it can be used for verification of numerical results if the upper bounds $\tilde{C}_{*} \geq c_{*}^{-1}$ and $\|L\|_{+} \geq\|L\|_{*}$ are at our disposal. In particular, $\tilde{C}_{*}$ defined by (3.13) can be used provided that the assumptions of Theorem 2.1 are satisfied. The bound $\|L\|_{+}$can be computed by techniques mentioned in [26]. In numerical examples presented Section 7, we shall determine $\|L\|_{+}$analytically.

\subsection{The equivalence between the static and kinematic approaches}

To prove $\lambda^{*}=\zeta^{*}$ under the assumptions (1.1), (4.2) and (4.6), we use the following alternative definitions of $\lambda^{*}$ and $\zeta^{*}[16]$ :

$$
\begin{gathered}
\zeta^{*}=\sup \left\{\lambda \geq 0 \mid \inf _{v \in \mathbb{V}}\left[\int_{\Omega} j_{\infty}(\varepsilon(v)) d x-\lambda L(v)\right]>-\infty\right\}, \\
\lambda^{*}=\sup \left\{\lambda \geq 0 \mid \inf _{v \in \mathbb{V}}\left[\int_{\Omega} j(\varepsilon(v)) d x-\lambda L(v)\right]>-\infty\right\},
\end{gathered}
$$

where

$$
j(e):=\sup _{\tau \in B}\left\{\tau: e-\frac{1}{2} \mathbb{C}^{-1} \tau: \tau\right\}, \quad e \in \mathbb{M}_{\text {sym }}^{d \times d},
$$

and $\mathbb{C}$ is an arbitrary linear, symmetric and elliptic mapping from $\mathbb{M}_{\text {sym }}^{d \times d}$ to $\mathbb{M}_{\text {sym }}^{d \times d}$. If the tensor $\mathbb{C}$ represents the Hooke law in elasticity then the inner inf-problem in (5.6) coincides with the Hencky problem $[16,36]$. For the sake of simplicity, we shall assume that $\mathbb{C}$ is the identity mapping from $\mathbb{M}_{\text {sym }}^{d \times d}$ to $\mathbb{M}_{\text {sym }}^{d \times d}$, i.e.,

$$
j(e):=\sup _{\tau \in B}\left\{\tau: e-\frac{1}{2}|\tau|^{2}\right\}, \quad e \in \mathbb{M}_{\text {sym }}^{d \times d} .
$$

Theorem 5.2. Let (1.1), (4.2) and (4.6) be satisfied. Then $\lambda^{*}=\zeta^{*}$.

Proof. Denote

$$
j^{\mathcal{C}}(e):=\sup _{\tau \in \mathcal{C}}\left\{\tau: e-\frac{1}{2}|\tau|^{2}\right\}, \quad j^{A}(e):=\sup _{\tau \in A}\left\{\tau: e-\frac{1}{2}|\tau|^{2}\right\}, \quad e \in \mathbb{M}_{\text {sym }}^{d \times d} .
$$

Notice that from (3.4), it follows that

$$
j^{\mathcal{C}}(e)=\frac{1}{2}\left|\Pi_{\mathcal{C}}(e)\right|^{2} \quad \forall e \in \mathbb{M}_{\text {sym }}^{d \times d} .
$$

First, we show several auxiliary results for $j^{A}$ and $j^{C}$ which will be used in what follows. It holds:

$$
\begin{array}{ll}
- & j(e) \geq j^{\mathcal{C}}(e)+j^{A}(e) \quad \forall e \in \mathbb{M}_{\text {sym }}^{d \times d}, \\
- & j^{\mathcal{C}}(e)=0, \quad j^{A}(e) \leq j(e) \leq j^{A}(e)+\rho_{A}^{2} / 2 \quad \forall e \in \mathcal{C}^{-}, \\
- & j^{A}(e)-j^{A}(\eta) \leq \rho_{A}|e-\eta| \quad \forall e, \eta \in \mathbb{M}_{s y m}^{d \times d}
\end{array}
$$


where $\rho_{A}$ is from (4.8). Indeed, using that $\tau_{A}: \tau_{\mathcal{C}} \leq 0$ for any $\tau_{A} \in A \subset \mathcal{C}^{-}$and any $\tau_{\mathcal{C}} \in \mathcal{C}$, we have

$$
j(e) \stackrel{(4.6)}{=} \sup _{\substack{\tau_{A} \in A \\ \tau_{\mathcal{C}} \in \mathcal{C}}}\left\{\left(\tau_{A}+\tau_{\mathcal{C}}\right): e-\frac{1}{2}\left|\tau_{A}+\tau_{\mathcal{C}}\right|^{2}\right\} \geq \sup _{\tau_{A} \in A}\left\{\tau_{A}: e-\frac{1}{2}\left|\tau_{A}\right|^{2}\right\}+\sup _{\tau_{\mathcal{C}} \in \mathcal{C}}\left\{\tau_{\mathcal{C}}: e-\frac{1}{2}\left|\tau_{\mathcal{C}}\right|^{2}\right\}
$$

for any $e \in \mathbb{M}_{\text {sym }}^{d \times d}$ proving (5.9).

Let $e \in \mathcal{C}^{-}$. Then $j^{\mathcal{C}}(e) \stackrel{(5.8)}{=} 0$. The first inequality in $(5.10)$ is obvious. Since $\tau_{\mathcal{C}}: e \leq 0$ for any $\tau_{\mathcal{C}} \in \mathcal{C}$ we obtain:

$$
\begin{aligned}
j(e) & \leq \sup _{\substack{\tau_{A} \in A \\
\tau_{\mathcal{C}} \in \mathcal{C}}}\left\{\tau_{A}: e-\frac{1}{2}\left|\tau_{A}\right|^{2}-\tau_{A}: \tau_{\mathcal{C}}-\frac{1}{2}\left|\tau_{\mathcal{C}}\right|^{2}\right\} \\
& \leq \sup _{\tau_{A} \in A}\left\{\tau_{A}: e-\frac{1}{2}\left|\tau_{A}\right|^{2}\right\}+\sup _{\substack{\tau_{A} \in A \\
\tau_{\mathcal{C}} \in \mathcal{C}}}\left\{-\tau_{A}: \tau_{\mathcal{C}}-\frac{1}{2}\left|\tau_{\mathcal{C}}\right|^{2}\right\} \\
& =j^{A}(e)+\sup _{\tau_{A} \in A} \frac{1}{2}\left|\Pi_{\mathcal{C}}\left(-\tau_{A}\right)\right|^{2} \leq j^{A}(e)+\frac{1}{2} \rho_{A}^{2} \quad \forall e \in \mathcal{C}^{-},
\end{aligned}
$$

making use of boundedness of $A$ and the inequality $\left|\Pi_{\mathcal{C}}\left(-\tau_{A}\right)\right| \leq\left|\tau_{A}\right|$. Hence, (5.10) holds.

It remains to verify (5.11):

$$
j^{A}(e)-j^{A}(\eta) \leq \sup _{\tau \in A}(e-\eta): \tau=j_{\infty}^{A}(e-\eta) \stackrel{(4.8)}{\leq} \rho_{A}|e-\eta| \quad \forall e, \eta \in \mathbb{M}_{s y m}^{d \times d} .
$$

From (4.7), the definitions of $j$ and $j_{\infty}$, we obtain the following two-sided bounds:

$$
j_{\infty}(e)-\frac{1}{2} \sup _{\tau \in A}|\tau|^{2} \leq j^{A}(e) \stackrel{(5.10)}{\leq} j(e) \leq j_{\infty}(e) \quad \forall e \in \mathcal{C}^{-} .
$$

This makes it possible to write (5.5) in the following equivalent form:

$$
\zeta^{*}=\sup \left\{\lambda \geq 0 \mid \inf _{w \in \mathcal{K}}\left[\int_{\Omega} j(\varepsilon(w)) d x-\lambda L(w)\right]>-\infty\right\} .
$$

Denote $J_{\lambda}(v):=\int_{\Omega} j(\varepsilon(v)) d x-\lambda L(v), v \in \mathbb{V}$.

Let $v \in \mathbb{V}$ be fixed. In view of (1.1) there exists $w_{v} \in \mathcal{K}$ such that the distance estimate (5.4) holds. Further,

$$
\begin{aligned}
& J_{\lambda}\left(w_{v}\right)=\int_{\Omega} j\left(\varepsilon\left(w_{v}\right)\right) d x-\lambda L\left(w_{v}\right) \stackrel{(5.10)}{\leq} \int_{\Omega} j^{A}\left(\varepsilon\left(w_{v}\right)\right) d x-\lambda L\left(w_{v}\right)+\frac{1}{2} \rho_{A}^{2}|\Omega| \\
& \stackrel{(5.9)}{\leq} \quad J_{\lambda}(v)-\int_{\Omega} j^{\mathcal{C}}(\varepsilon(v)) d x+\int_{\Omega}\left[j^{A}\left(\varepsilon\left(w_{v}\right)\right)-j^{A}(\varepsilon(v))\right] d x \\
& +\lambda L\left(v-w_{v}\right)+\frac{1}{2} \rho_{A}^{2}|\Omega| \\
& \stackrel{(5.8),(5.11)}{\leq} J_{\lambda}(v)-\frac{1}{2}\left\|\Pi_{\mathcal{C}} \varepsilon(v)\right\|_{\Omega}^{2}+\int_{\Omega} \rho_{A}\left|\varepsilon\left(v-w_{v}\right)\right| d x \\
& +\lambda\|L\|_{*}\left\|\nabla\left(v-w_{v}\right)\right\|_{\Omega}+\frac{1}{2} \rho_{A}^{2}|\Omega| \\
& \leq \quad J_{\lambda}(v)-\frac{1}{2}\left\|\Pi_{\mathcal{C}} \varepsilon(v)\right\|_{\Omega}^{2}+\left(\rho_{A}|\Omega|^{1 / 2}+\lambda\|L\|_{*}\right)\left\|\nabla\left(v-w_{v}\right)\right\|_{\Omega}+\frac{1}{2} \rho_{A}^{2}|\Omega| \\
& \stackrel{(5.4)}{\leq} \quad J_{\lambda}(v)-\frac{1}{2}\left\|\Pi_{\mathcal{C}} \varepsilon(v)\right\|_{\Omega}^{2}+c_{*}^{-1}\left(\rho_{A}|\Omega|^{1 / 2}+\lambda\|L\|_{*}\right)\left\|\Pi_{\mathcal{C}} \varepsilon(v)\right\|_{\Omega}+\frac{1}{2} \rho_{A}^{2}|\Omega| \\
& \leq \quad J_{\lambda}(v)+\frac{\left(\rho_{A}|\Omega|^{1 / 2}+\lambda\|L\|_{*}\right)^{2}}{2 c_{*}^{2}}+\frac{1}{2} \rho_{A}^{2}|\Omega| .
\end{aligned}
$$


Consequently,

$$
\inf _{v \in \mathbb{V}} J_{\lambda}(v) \leq \inf _{w_{v} \in \mathcal{K}} J_{\lambda}\left(w_{v}\right) \leq \inf _{v \in \mathbb{V}} J_{\lambda}(v)+\frac{\left(\rho_{A}|\Omega|^{1 / 2}+\lambda\|L\|_{*}\right)^{2}}{2 c_{*}^{2}}+\frac{1}{2} \rho_{A}^{2}|\Omega| \quad \forall \lambda \geq 0,
$$

so that $\lambda^{*}=\zeta^{*}$ as follows from (5.13) and (5.6).

\section{$6 \quad$ Examples of yield criteria}

In this section, we apply the abstract results of Sections 2-5 to the von Mises and Drucker-Prager yield criteria.

\subsection{The von Mises yield criterion}

The set $B$ corresponding to the von Mise yield criterion is defined as follows:

$$
B:=\left\{\tau \in \mathbb{M}_{\text {sym }}^{d \times d}|| \tau^{D} \mid \leq \gamma\right\}
$$

where $\gamma>0$ is a material parameter representing the yield stress. This set is an unbounded cylinder aligned with the hydrostatic axis $\mathcal{C}=\left\{\tau \in \mathbb{M}_{\text {sym }}^{d \times d} \mid \tau=q I, \quad q \in \mathbb{R}\right\}$, see [13]. From Section 1, we known that the inf-sup condition (1.1) holds for such $\mathcal{C}$ and is equivalent to (1.2).

Further, the polar cone $\mathcal{C}^{-}$to $\mathcal{C}$ coincides with the orthogonal complement $\mathcal{C}^{\perp}$ of $\mathcal{C}$ in $\mathbb{M}_{\text {sym }}^{d \times d}$ :

$$
\mathcal{C}^{-}=\mathcal{C}^{\perp} \stackrel{(2.3)}{=}\left\{\tau \in \mathbb{M}_{\text {sym }}^{d \times d} \mid \operatorname{tr} \tau=0\right\}=\left\{\tau \in \mathbb{M}_{\text {sym }}^{d \times d} \mid \tau=\tau^{D}\right\}
$$

and

$$
B=\mathcal{C}+A, \quad A=B \cap \mathcal{C}^{-}=\left\{\tau \in \mathcal{C}^{-}|| \tau \mid \leq \gamma\right\}
$$

Consequently, the assumptions (4.2) and (4.6) are satisfied, $j_{\infty}^{A}(e)=\max _{\tau \in A} \tau: e=\gamma\left|e^{D}\right|$ for any $e \in \mathbb{M}_{\text {sym }}^{d \times d}$ and the limit analysis problem (4.10) reads as

$$
\zeta^{*}=\gamma \inf _{\substack{v \in \mathcal{K} \\ L(v)=1}} \int_{\Omega}\left|\varepsilon^{D}(v)\right| d x, \quad \mathcal{K}=\{w \in \mathbb{V} \mid \operatorname{div} w=0 \text { a.e. in } \Omega\}
$$

Since the projection $\Pi_{\mathcal{C}}$ defined by (3.2) satisfies

$$
\Pi_{\mathcal{C}} e \stackrel{(2.3)}{=} \frac{1}{d}(\operatorname{tr} e) I, \quad\left|\Pi_{\mathcal{C}} e\right|^{2}=\frac{1}{d}(\operatorname{tr} e)^{2} \quad \forall e \in \mathbb{M}_{\text {sym }}^{d \times d}
$$

Theorem 3.1 ensures the following distance estimate:

$$
\min _{w \in \mathcal{K}}\|\nabla(v-w)\|_{\Omega} \leq C_{\Omega}\|\operatorname{div} v\|_{\Omega} \quad \forall v \in \mathbb{V}
$$

where $C_{\Omega}^{-1}=c_{\Omega}$ is the inf-sup constant from (1.2). In view of (6.1) one can use the results of Theorems 5.1 and 5.2 for the von Mises yield criterion, see also [31]. These results may be extended to $\Gamma_{0}=\partial \Omega$. In this case, the distance estimate (6.1) holds with the constant $C_{\Omega_{\widetilde{C}}}^{0}$ defined by (2.9), see $[2,18,25,26]$. To be the bounds computable, one can replace $C_{\Omega}^{0}, C_{\Omega}$ by $\tilde{C}_{\Omega}^{0}, \tilde{C}_{\Omega}$ defined by $(2.11)$, and (2.12), respectively.

Remark 6.1. Analogous results may be also derived for the Tresca yield criterion $[13,36]$ since the cone $\mathcal{C}$ is the same for both, the von Mises and Tresca yield criteria. 


\subsection{The Drucker-Prager yield criterion}

The set $B$ corresponding to the Drucker-Prager yield criterion is defined by

$$
B:=\left\{\tau \in \mathbb{M}_{\text {sym }}^{d \times d}|| \tau^{D} \mid+\frac{a}{d} \operatorname{tr} \tau \leq \gamma\right\}, \quad a, \gamma>0 .
$$

It is the cone with the vertex at $\frac{\gamma}{a} I$ (see [16]). We have:

$$
\begin{gathered}
B=\mathcal{C}+\left\{\frac{\gamma}{a} I\right\}, \quad \mathcal{C}=\left\{\tau \in \mathbb{M}_{\text {sym }}^{d \times d}|| \tau^{D} \mid+\frac{a}{d} \operatorname{tr} \tau \leq 0\right\}, \\
\mathcal{C}^{-}=\left\{\eta \in \mathbb{M}_{\text {sym }}^{d \times d}|\operatorname{tr} \eta \geq a| \eta^{D} \mid\right\} .
\end{gathered}
$$

Indeed, one can easily verify the polarity between $\mathcal{C}$ and $\mathcal{C}^{-}$:

$$
\tau: \eta=\tau^{D}: \eta^{D}+\frac{1}{d}(\operatorname{tr} \tau)(\operatorname{tr} \eta) \leq \tau^{D}: \eta^{D}-\left|\tau^{D}\right|\left|\eta^{D}\right| \leq 0 \quad \forall \tau \in \mathcal{C}, \forall \eta \in \mathcal{C}^{-} .
$$

Notice that $\frac{\gamma}{a} I \in A=B \cap \mathcal{C}^{-}$and $B=\mathcal{C}+\left\{\frac{\gamma}{a} I\right\}=\mathcal{C}+A$. Since $A$ is bounded, the assumptions (4.2) and (4.6) are satisfied and

$$
j_{\infty}^{A}(e)=\max _{\tau \in A} \tau: e=\frac{\gamma}{a} \operatorname{tr} e \quad \forall e \in \mathbb{M}_{\text {sym }}^{d \times d}, \quad \mathcal{K}=\left\{w \in \mathbb{V} \mid \operatorname{div} w \geq a \varepsilon^{D}(w) \text { a.e. in } \Omega\right\} .
$$

Thus the kinematic limit analysis problem (4.10) reads as

$$
\zeta^{*}=\inf _{\substack{v \in \mathcal{K} \\ L(v)=1}} J_{\infty}^{A}(v), \quad J_{\infty}^{A}(v)=\frac{\gamma}{a} \int_{\Omega} \operatorname{div} v d x .
$$

Further, the inf-sup condition (1.1) is valid under the additional assumption on the material parameter $a$ defining the slope of the Drucker-Prager cone:

$$
a<\left(\tilde{C}_{\Omega}^{2}-d^{-1}\right)^{-1 / 2},
$$

where $\tilde{C}_{\Omega}>0$ is the constant from (2.12). Indeed, from (6.3), it follows that the assumption (2.4) of Theorem 2.1 holds with $\hat{a}=a / d$. We arrive at the following consequences of Theorems 2.1, 3.1, 5.1, and 5.2 .

Corollary 6.1. Let $a>0$ satisfy (6.3). Then

$$
\begin{gathered}
c_{*}=\inf _{\substack{\tau \in L^{2}(\Omega ; \mathcal{C}) \\
\tau \neq 0}} \sup _{\substack{v \in \mathbb{V} \\
v \neq 0}} \frac{\int_{\Omega} \tau: \varepsilon(v) d x}{\|\tau\|_{\Omega}\|\nabla v\|_{\Omega}} \geq \frac{1-a \sqrt{\tilde{C}_{\Omega}^{2}-d^{-1}}}{\tilde{C}_{\Omega} \sqrt{a^{2}+d}}>0, \\
\min _{w \in \mathcal{K}}\|\nabla(v-w)\|_{\Omega} \leq C_{D P}\left\|\Pi_{\mathcal{C}} \varepsilon(v)\right\|_{\Omega} \quad \forall v \in \mathbb{V}, \quad C_{D P}:=\frac{\tilde{C}_{\Omega} \sqrt{a^{2}+d}}{1-a \sqrt{\tilde{C}_{\Omega}^{2}-d^{-1}}},
\end{gathered}
$$

and

$$
\lambda^{*}=\zeta^{*} \leq \frac{J_{\infty}^{A}(v)+\rho_{A} C_{D P}|\Omega|^{1 / 2}\left\|\Pi_{\mathcal{C}} \varepsilon(v)\right\|_{\Omega}}{L(v)-C_{D P}\|L\|_{+}\left\|\Pi_{\mathcal{C}} \varepsilon(v)\right\|_{\Omega}},
$$

holds for any $v \in \mathbb{V}$ such that $L(v)>C_{D P}\|L\|_{+}\left\|\Pi_{\mathcal{C}} \varepsilon(v)\right\|_{\Omega}$, where

$$
\rho_{A}=\max _{\tau \in A}|\tau|=\frac{\gamma}{\alpha} \sqrt{d}, \quad\|L\|_{+} \geq\|L\|_{*}=\sup _{\substack{v \in \mathbb{V} \\ v \neq 0}} \frac{L(v)}{\|\nabla v\|_{\Omega}} .
$$


Remark 6.2. In practice, $a>0$ depends on the friction angle (see below) and cannot be arbitrarily large. In addition, for larger $a$, one can expect that the limit values will be equal to $+\infty$ as follows from $[23,24,30]$. Despite these facts, the assumption (6.3) seems to be restrictive since the material parameter $a$ is related to the constant $\tilde{C}_{\Omega}$ which depends only on the shape of the domain $\Omega$.

Remark 6.3. The equality $\zeta^{*}=\lambda^{*}$ for the Drucker-Prager yield criterion was originally proven for sufficiently small $a>0$ in [30, Appendix 1] but without any bound on $a>0$. From [30], it is also known that $\lambda^{*}=\zeta^{*}=+\infty$ if $\mathbb{V}=W_{0}^{1,2}\left(\Omega ; \mathbb{R}^{d}\right)$. In this case, $\mathcal{K}=\{0\}$ by Remark 3.2 since int $\mathcal{C} \neq \emptyset$ in $\mathbb{M}_{s y m}^{d \times d}$.

Remark 6.4. Let us note that the crucial assumption (2.4) can be also verified for the MohrCoulomb yield criterion because the Mohr-Coulomb yield surface can be estimated from both sides by the Drucker-Prager yield surface, see, e.g., [13].

For computations, the closed form of the projection $\Pi_{\mathcal{C}}$ defined by (3.1) is needed.

Lemma 6.1. It holds:

$$
\begin{aligned}
& \Pi_{\mathcal{C}} e=\left\{\begin{array}{cl}
e, & e \in \mathcal{C}, \\
0, & e \in \mathcal{C}^{-}, \\
\frac{a\left|e^{D}\right|-\operatorname{tr} e}{d+a^{2}}\left(a \frac{e^{D}}{\left|e^{D}\right|}-I\right), & e \notin \mathcal{C} \cup \mathcal{C}^{-},
\end{array}\right. \\
& \left|\Pi_{\mathcal{C}} e\right|^{2}=\left\{\begin{array}{cl}
|e|^{2}, & e \in \mathcal{C}, \\
0, & e \in \mathcal{C}^{-}, \\
\frac{1}{d+a^{2}}\left(a\left|e^{D}\right|-\operatorname{tr} e\right)^{2}, & e \notin \mathcal{C} \cup \mathcal{C}^{-} .
\end{array}\right.
\end{aligned}
$$

Proof. (sketch) It is sufficient to prove $(6.6)_{3}$. Let $e \notin \mathcal{C} \cup \mathcal{C}^{-}$and denote $\tau_{e}:=\Pi_{\mathcal{C}} e$. Then $\tau_{e} \in \partial \mathcal{C}$ and $\left|\tau_{e}^{D}\right|>0$. Using the Karush-Kuhn-Tucker conditions, there exists a multiplier $\mu>0$ such that

$$
e-\tau_{e}=\mu\left(\frac{\tau_{e}^{D}}{\left|\tau_{e}^{D}\right|}+\frac{a}{d} I\right), \quad\left|\tau_{e}^{D}\right|+\frac{a}{d} \operatorname{tr} \tau_{e}=0 .
$$

By the decomposition of tensors into the deviatoric and volumetric parts, we arrive at

$$
\operatorname{tr}\left(e-\tau_{e}\right)=\mu a, \quad \frac{\tau_{e}^{D}}{\left|\tau_{e}^{D}\right|}=\frac{e^{D}}{\left|e^{D}\right|}, \quad\left|\tau_{e}^{D}\right|=\left|e^{D}\right|-\mu, \quad\left|\tau_{e}^{D}\right|+\frac{a}{d} \operatorname{tr} \tau_{e}=0 .
$$

Hence, it is easy to show that

$$
\mu=\frac{d}{d+a^{2}}\left(\left|e^{D}\right|+\frac{a}{d} \operatorname{tr} e\right)
$$

and

$$
\Pi_{\mathcal{C}} e=\tau_{e}=e-\mu\left(\frac{e^{D}}{\left|e^{D}\right|}+\frac{a}{d} I\right)=\frac{a\left|e^{D}\right|-\operatorname{tr} e}{d+a^{2}}\left(a \frac{e^{D}}{\left|e^{D}\right|}-I\right) .
$$

From (6.6), one can easily obtain (6.7).

\section{Numerical examples}

In this section, we consider strip-footing and slope stability problems with the Drucker-Prager yield criterion, see e.g. $[9,13]$. These problems are formulated under the plane strain assumptions enabling us to use a dimensional reduction with $\Omega \subset \mathbb{R}^{2}$. On the other hand, $B$ is a subset of $\mathbb{M}_{\text {sym }}^{3 \times 3}$ and thus $d=3$ in the definition of the constant $C_{D P}$ used in (6.4). First, we briefly summarize our computational strategy for kinematic limit analysis which has been systematically developped in $[34,7,15,16,33,31]$. Unlike these papers, we also use a simple local mesh adaptivity in order to improve numerical results. For other computational techniques in limit analysis, we refer to $[4,6,12,10,16,17,19,32,37]$. 


\subsection{Computational strategy}

We solve the problem $(\mathcal{P})^{\infty}$ defined by $(4.4)$ or (6.2) and compute the related upper bound by penalization and conforming finite elements. The penalized problem reads as

$$
(\mathcal{P})^{\alpha} \quad \inf _{\substack{v \in \mathbb{V} \\ L(v)=1}} \int_{\Omega} j_{\alpha}(\varepsilon(v)) d x, \quad j_{\alpha}(e)=\sup _{\tau \in B}\left\{\tau: e-\frac{1}{2 \alpha} \mathbb{C}^{-1} \tau: \tau\right\}, \quad e \in \mathbb{M}_{\text {sym }}^{d \times d},
$$

where $\alpha>0$ is the penalization parameter and $\mathbb{C}$ is an arbitrary symmetric and positive definite fourth order tensor. It holds that $j_{\alpha}$ is convex, smooth and $j_{\alpha} \rightarrow j_{\infty}$ in $\mathbb{M}_{s y m}^{d \times d}$ as $\alpha \rightarrow+\infty$. Further, if $\mathbb{C}$ is the elasticity tensor then the penalized problem is closely related to a static version of the elastic-perfectly plastic problem, see $[15,16,33]$. Let $\mathbb{V}_{h}$ be a finite-dimensional subspace of $\mathbb{V}$ and $(\mathcal{P})_{h}^{\alpha}$ denote the discrete counterpart of the problem $(\mathcal{P})^{\alpha}$. Such a problem has a solution denoted as $u_{h, \alpha}$. We introduce two auxiliary functions depending on $\alpha$.

The first function is defined by

$$
\psi_{h}(\alpha):=\int_{\Omega} \Pi_{B}\left(\varepsilon\left(\alpha u_{h, \alpha}\right)\right): \varepsilon\left(u_{h, \alpha}\right) d x, \quad \alpha>0
$$

where $\Pi_{B}$ is the projection of $\mathbb{M}_{s y m}^{d \times d}$ onto $B$ w.r.t. the biscalar product. This function has been introduced and analyzed in $[15,16,33,31]$ and we summarize its properties. It holds that $j_{\alpha}^{\prime}(e)=$ $\Pi_{B}(\alpha e)$ for any $\alpha>0, e \in \mathbb{M}_{\text {sym }}^{d \times d}$ and $\Pi_{B}$ represents the projection of $\mathbb{M}_{\text {sym }}^{d \times d}$ onto $B$. We know that $u_{h, \alpha}$ is also a solution to the discrete elastic-perfectly plastic problem with the load parameter $\lambda_{\alpha}:=\psi_{h}(\alpha)$. Further, $\psi_{h}$ is a continuous and nondecreasing function satisfying $\psi_{h}(0)=0, \lim _{\alpha \rightarrow+\infty} \psi_{h}(\alpha)=\lambda_{h}^{*}=$ $\zeta_{h}^{*}$ where $\lambda_{h}^{*}, \zeta_{h}^{*}$ are the discrete counterparts to $\lambda^{*}$, and $\zeta^{*}$, respectively. If we consider a regular system of finite element partitions of $\bar{\Omega}$ then $\psi_{h}(\alpha) \rightarrow \psi(\alpha)$ as $h \rightarrow 0_{+}$for any $\alpha>0$. The limit function $\psi: \mathbb{R}_{+} \rightarrow \mathbb{R}_{+}$is also continuous, nondecreasing and $\psi(\alpha) \rightarrow \lambda^{*}$ as $\alpha \rightarrow+\infty$. Hence, $\lambda_{h}^{*} \geq \lambda^{*} \geq \psi(\alpha)$ for any $\alpha>0$ and $h>0$. Convergence $\lambda_{h}^{*} \rightarrow \lambda^{*}$ need not hold. The mentioned properties of the functions $\psi_{h}$ and $\psi$ are depicted in Figure 2.

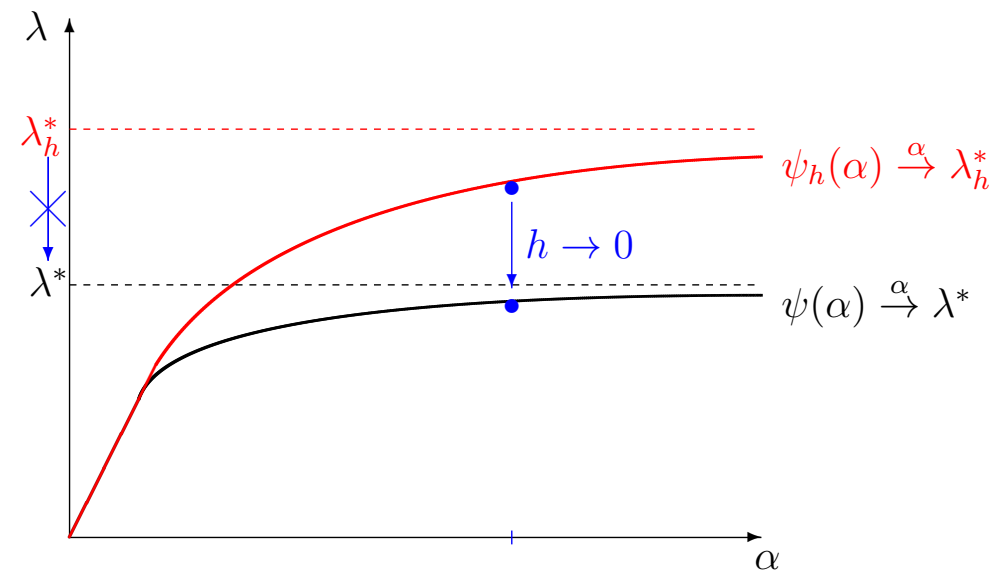

Figure 2: Properties of the functions $\psi_{h}$ and $\psi$.

The second function

$$
\Psi_{h}(\alpha):=\left\{\begin{array}{cc}
\frac{J_{\infty}^{A}\left(u_{h, \alpha}\right)+\rho_{A} C_{D P}|\Omega|^{1 / 2}\left\|\Pi_{\mathcal{C}} \varepsilon\left(u_{h, \alpha}\right)\right\|_{\Omega}}{L\left(u_{h, \alpha}\right)-C_{D P}\|L\|_{+}\left\|\Pi_{\mathcal{C}} \varepsilon\left(u_{h, \alpha}\right)\right\|_{\Omega}}, & \text { if } L\left(u_{h, \alpha}\right)>C_{D P}\|L\|_{+}\left\|\Pi_{\mathcal{C}} \varepsilon\left(u_{h, \alpha}\right)\right\|_{\Omega}, \\
+\infty, & \text { otherwise, }
\end{array}\right.
$$

arises from the guaranteed upper bound (6.4) with $v=u_{h, \alpha}$. This function was originally introduced and analyzed for the von Mises yield criterion in [31]. It is worth mentioning that the values of $\Psi_{h}$ are upper bounds of $\zeta^{*}$ but they need not be upper bounds of the discrete limit load $\lambda_{h}^{*}=\zeta_{h}^{*}$. 
Problem $(\mathcal{P})_{h}^{\alpha}$ is solved by the semismooth Newton method (SSNM). Since $(\mathcal{P})_{h}^{\alpha}$ is a minimization problem, SSNM is interpreted as a sequential quadratic programming. If $\alpha$ is large then $(\mathcal{P})_{h}^{\alpha}$ is strongly nonlinear. For this reason, SSNM is supplied with with damping and/or a continuation with respect to $\alpha$, see $[34,7,16]$. Due to continuation, one can find a sufficiently large value $\alpha_{\max }$ of $\alpha$ for which the functions $\psi_{h}$ and $\Psi_{h}$ are already almost constant.

In limit analysis, the failure is usually localized and rigid deformation fields are observed far from the failure. Therefore, we use local mesh adaptivity since it can significantly reduce the number of unknowns and improve accuracy of the results. For the sake of simplicity, we consider right-angled, isoscaled triangles (2D elements) before and after the refinement to avoid a mesh degeneration. The refined mesh is constructed by means of the bisection technique.

We use the following mesh adaptive strategy. First, the maximal value $\alpha_{\max }$ of $\alpha$ achieved by continuation on the coarsest mesh $\mathcal{T}_{h_{0}}$ is fixed. For the mesh level $k=0,1,2, \ldots$, we denote the solution to $(\mathcal{P})_{h_{k}}^{\alpha_{\max }}$ by $u_{k}$. Then $\int_{T} j_{\infty}^{A}\left(\varepsilon\left(u_{k}\right)\right) d x$ is evaluated for any $T \in \mathcal{T}_{h_{k}}$ and $10 \%$ of elements with the highest values is selected. This set of elements has to be slightly modified in order to obtain the $(k+1)$-th mesh level created by only right-angled triangles. For $k=1,2, \ldots$, problem $(\mathcal{P})_{h_{k}}^{\alpha_{\max }}$ is solved by damped SSNM without continuation. For better convergence, we use $u_{k-1}$ on the finer mesh to initiate Newton's method on the finer mesh.

The material parameters $a$ and $\gamma$ are usually computed from the cohesion $\left(c_{0}\right)$ and the friction angle $(\phi)$. For the plane strain problems, the following formulas are recommended [13, Chapter 6$]$ :

$$
a=\frac{3 \sqrt{2} \tan \phi}{\sqrt{9+12 \tan ^{2} \phi}}, \quad \gamma=c_{0} \frac{3 \sqrt{2}}{\sqrt{9+12 \tan ^{2} \phi}} .
$$

The problem is implemented in Matlab. Tangential stiffness matrices and load vectors are assembled by vectorized codes described in [8]. These codes are available for P1, P2, Q1 and Q2 elements in 2D and 3D. Numerical examples presented below use P2 elements with the 7-point Gauss quadrature for numerical integration on triangular elements.

\subsection{The strip-footing problem}

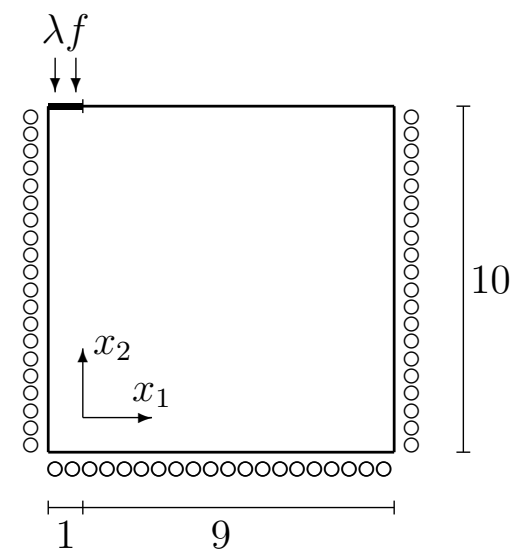

Figure 3: Geometry of the strip-footing problem.

The first example is a strip-footing problem in which the bearing capacity of a soil foundation is analyzed. 2D geometry of the plane strain problem is depicted in Figure 3. On the left, right and bottom sides of the square domain, the zero normal displacements are prescribed, i.e.

$$
\mathbb{V}=\left\{v=\left(v_{1}, v_{2}\right) \in W^{1,2}\left(\Omega ; \mathbb{R}^{2}\right) \mid v_{1}\left(0, x_{2}\right)=v_{1}\left(10, x_{2}\right)=0, v_{2}\left(x_{1}, 0\right)=0, x_{1}, x_{2} \in(0,10)\right\} .
$$

The condition on the left vertical side is due to the symmetry of the problem. The strip-footing of the length one is considered on the top of the domain. For the sake of simplicity, we apply 
there the normal pressure of density $\lambda f$, where $\lambda \geq 0$ is the load parameter and $f=-450$, i.e., $L(v)=-450 \int_{0}^{1} v_{2}\left(x_{1}, 10\right) d x_{1}, v=\left(v_{1}, v_{2}\right)$. Further, the values $c_{0}=450$ and $\phi=\pi / 18$ giving $a \doteq 0.2444$ and $\gamma \doteq 623.6$ are used. We are interested in the limit value $\lambda^{*}$ of $\lambda$.

In order to use the guaranteed upper bound (6.4), one has to estimate the constants $C_{D P}$ and $\|L\|_{+}$. We have:

$$
\begin{aligned}
L(v) & =-450 \int_{0}^{1} v_{2}\left(x_{1}, 10\right) d x_{1}=-450 \int_{0}^{1}\left[v_{2}\left(x_{1}, 10\right)-v_{2}\left(x_{1}, 0\right)\right] d x_{1} \\
& =-450 \int_{0}^{1} \int_{0}^{10} \frac{\partial v_{2}}{\partial x_{2}} d x_{2} d x_{1} \leq 450 \sqrt{10}\|\nabla v\|_{\Omega} \quad \forall v \in \mathbb{V} .
\end{aligned}
$$

Hence, $\|L\|_{+} \doteq 450 \sqrt{10}$. From $(2.11),(2.12)$ and Corollary 6.1, we obtain

$$
C_{D P}=\frac{\tilde{C}_{\Omega} \sqrt{a^{2}+3}}{1-a \sqrt{\tilde{C}_{\Omega}^{2}-1 / 3}}, \quad \tilde{C}_{\Omega}=\sqrt{\left(\tilde{C}_{\Omega}^{0}\right)^{2}+|\Omega|^{-1}\|\nabla \tilde{v}\|_{\Omega}^{2}}, \quad \tilde{C}_{\Omega}^{0}:=\frac{\sqrt{2}}{\kappa}\left(1+\sqrt{1-\kappa^{2}}\right)^{1 / 2},
$$

where $\kappa=\rho / R=1 / \sqrt{2}$ and $\tilde{v} \in \mathbb{V}$ satisfies $\operatorname{div} \tilde{v}=1$. The choice $\tilde{v}=\left(0, x_{2}\right)$ leads to $|\Omega|^{-1}\|\nabla \tilde{v}\|_{\Omega}^{2}=1$. Hence, $\tilde{C}_{\Omega}^{0} \doteq 2.6131, \tilde{C}_{\Omega} \doteq 2.7979$, and $C_{D P} \doteq 14.7839$. In view of (6.3), it follows that $\lambda^{*}=\zeta^{*}$.

The numerical results are depicted in Figures 4-6. In Figure 4, we compare the values $\psi_{h}(\alpha)$ and $\Psi_{h}(\alpha)$ for different mesh levels. The figure on the left corresponds to the coarsest mesh where the continuation with respect to $\alpha$ was used. We observe that the values $\psi_{h}(\alpha), \alpha>10^{10}$, are practically constant and approximate the discrete limit load $\lambda_{h}^{*}$. We see that the upper bound function $\Psi_{h}$ is decreasing and the values $\Psi_{h}(\alpha)$ overestimate $\lambda^{*}$ for $\alpha$ small. Therefore, we set $\alpha_{\max } \approx 10^{12}$ for which the values of $\psi_{h}$ and $\Psi_{h}$ close to each other. Then the local mesh adaptivity for $\alpha=\alpha_{\max }$ with 37 mesh levels is used, see the figure on the right. During the mesh refinement, the bounds of $\lambda^{*}$ are reduced. We see that the difference between $\psi_{h}\left(\alpha_{\max }\right)$ and $\Psi_{h}\left(\alpha_{\max }\right)$ is negligible and it remains almost constant. In particular, $\psi_{h}\left(\alpha_{\max }\right) \doteq 8.35$ and $\Psi_{h}\left(\alpha_{\max }\right) \doteq 8.36$. Since $\lim _{h \rightarrow 0_{+}} \psi_{h}\left(\alpha_{\max }\right)=\psi\left(\alpha_{\max }\right) \leq \lambda^{*}$, one can expect that the lower bound of $\lambda^{*}$ is also close to 8.3.

The finest mesh and its detail are depicted in Figure 5. It consists of 13695 elements and 27525 nodes. We see that the mesh was refined only in a vicinity of footing. On the remaining part of the domain, the original (coarsest) mesh is preserved. The finest mesh corresponds to the failure visualized in Figure 6 (in a vicinity of the footing). Here, $\left|u_{h, \alpha_{\max }}\right|$ (left) and div $u_{h, \alpha_{\max }}$ (right) are depicted. The deformed shape on the left is enlarged for better visualization. We observe a significant jump of $u_{h, \alpha_{\max }}$ on the interface between the "dark" and "light" regions. This interface estimates the expected failure (slip) surface. The values practically vanish in the dark region.

\subsection{The slope stability problem}

The second example deals with the slope stability problem which is depicted in Figure 7 . The slope inclination is 45 degrees. The body is fixed on the left, bottom and right sides, i.e.,

$$
\mathbb{V}=\left\{v \in W^{1,2}\left(\Omega ; \mathbb{R}^{2}\right) \mid v\left(0, x_{2}\right)=v\left(10, x_{2}\right)=v\left(x_{1}, 0\right)=0, x_{1}, x_{2} \in(0,10)\right\} .
$$

We prescribe the gravitation force of density $\lambda F$, where $\lambda \geq 0$ is the load parameter and $F=-20$, i.e. $L(v)=-20 \int_{\Omega} v_{2} d x, v=\left(v_{1}, v_{2}\right)$. We set $c_{0}=50$ and $\phi=\pi / 18$ and so $a \doteq 0.2444, \gamma \doteq 69.2891$. We are interested in the limit value $\lambda^{*}$ of $\lambda$ and its upper bound.

In order to use the guaranteed upper bound (6.4), we have to estimate the constants $\|L\|_{+}$and $C_{D P}$. Since

$$
\begin{aligned}
L(v) & =-20 \int_{\Omega} v_{2} d x=-20 \int_{\Omega}\left[v_{2}\left(x_{1}, x_{2}\right)-v_{2}\left(x_{1}, 0\right)\right] d x \\
& =-20 \int_{\Omega} \int_{0}^{x_{2}} \frac{\partial v_{2}}{\partial x_{2}} d x_{2} d x \leq \frac{200}{3}\|\nabla v\|_{\Omega} \quad \forall v \in \mathbb{V}
\end{aligned}
$$



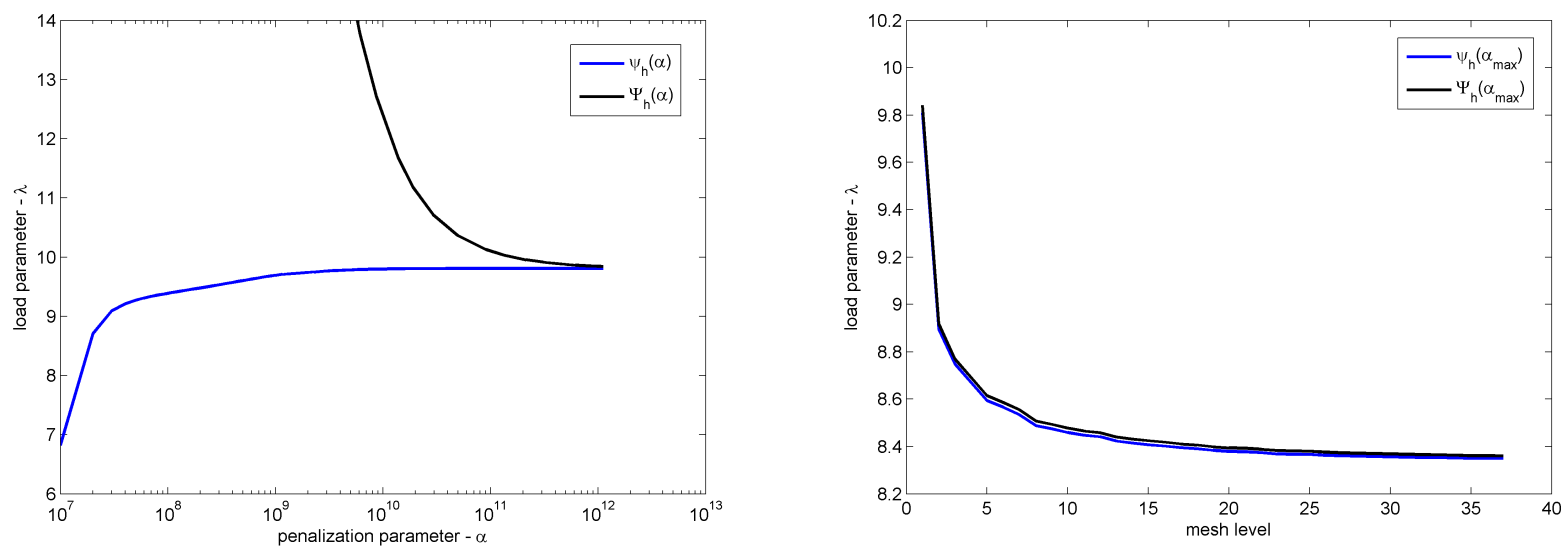

Figure 4: Comparison of $\psi_{h}$ and $\Psi_{h}$ for the strip-footing problem: left $-\alpha$-convergence for the initial-coarsest mesh; right - values $\psi_{h}\left(\alpha_{\max }\right)$ and $\Psi_{h}\left(\alpha_{\max }\right)$ depending on the mesh density.
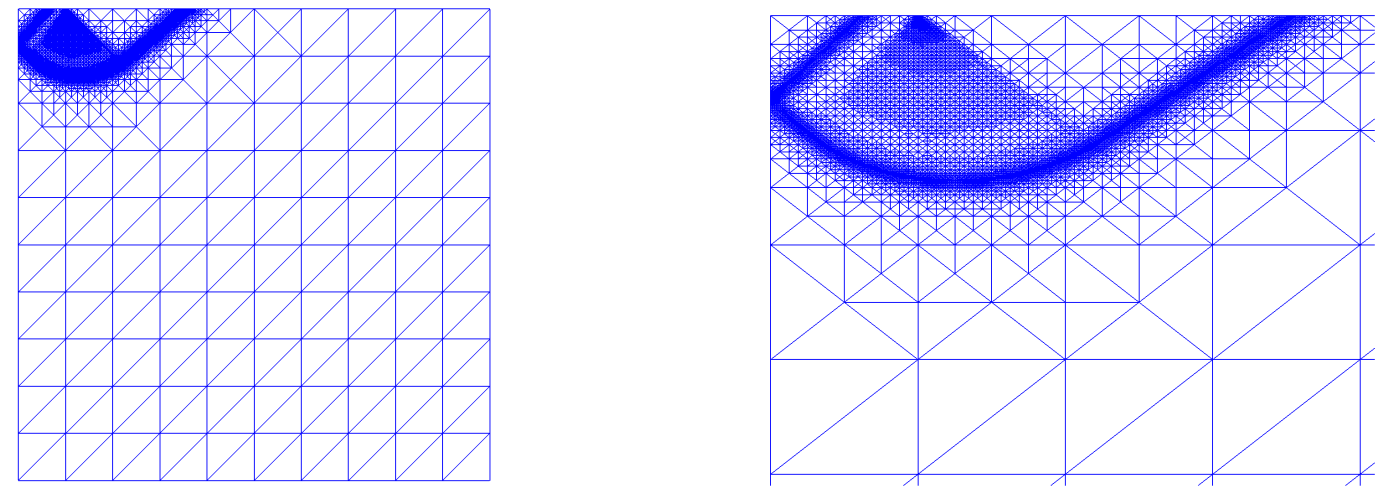

Figure 5: The finest mesh and its detail.
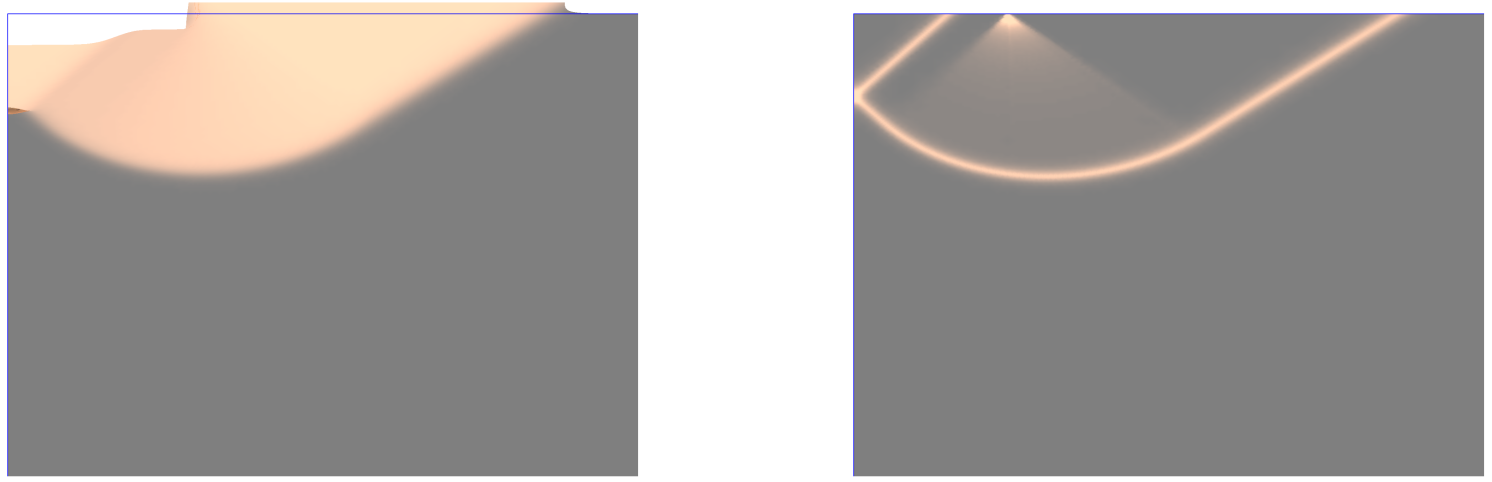

Figure 6: The field $u_{h, \alpha_{\max }}$ with enlarged deformed shapes (left) and the divergence of $u_{h, \alpha_{\max }}$ (right) for the finest mesh - strip footing problem. 


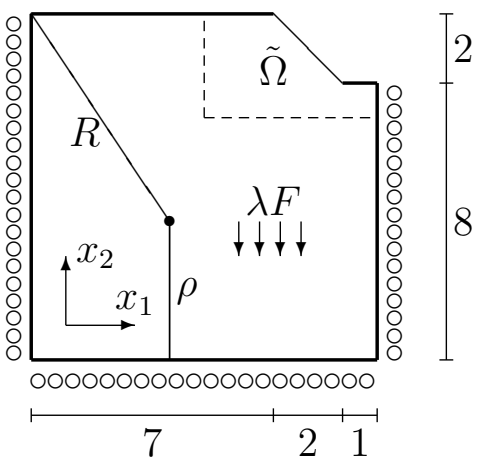

Figure 7: Geometry of the slope stability problem.

we have $\|L\|_{+} \doteq 66.67$. The bounds $\tilde{C}_{\Omega}^{0}, \tilde{C}_{\Omega}$ and $C_{D P}$ are computed by the same formulas as in the previous example. In particular, for evaluation of $\tilde{C}_{\Omega}^{0}$ and $\tilde{C}_{\Omega}$, we use $\kappa=\rho / R$ with the constants $R$ and $\rho$ depicted in Figure 7 and the function $\tilde{v}=\left(0, x_{2}\right)$. We obtain $\tilde{C}_{\Omega}^{0} \doteq 3.4508, \tilde{C}_{\Omega} \doteq 3.5928$, $C_{D P} \doteq 47.2226$, and again $\lambda^{*}=\zeta^{*}$ making use of Corollary 6.1.

Notice that the parts of $\partial \Omega$, which are far from the slope, are artifical. The chosen geometry in Figure 7 is non-standard but it enabled to reduce the ratio $\kappa=\rho / R$ and thus to improve the bound $C_{D P}$. Otherwise, we are not able to determine a sufficienty sharp upper bound of $\zeta^{*}$. Nevertheless, for computation, we shall only use functions from the following subspace of $\mathbb{V}$ :

$$
\tilde{\mathbb{V}}:=\{v \in \mathbb{V} \mid v=0 \text { in } \Omega \backslash \tilde{\Omega}\}, \quad \tilde{\Omega}:=\Omega \cap[(5,10) \times(7,10)],
$$

and solve the auxiliary problem

$$
\tilde{\zeta}^{*}=\inf _{\substack{\tilde{v} \tilde{\mathcal{K}} \\ L(\tilde{v})=1}} J_{\infty}^{A}(\tilde{v}), \quad \tilde{\mathcal{K}}:=\mathcal{K} \cap \tilde{\mathbb{V}} .
$$

Clearly, $\zeta^{*} \leq \tilde{\zeta}^{*}$ and this problem can be solved only on the subdomain $\tilde{\Omega}$ depicted in Figure 7 . Since it is observed that the kinematic minimizer in (6.2) vanishes far from the slope $[9,13]$, one can even $\operatorname{expect} \zeta^{*}=\tilde{\zeta}^{*}$.

Numerical results are depicted in Figures $8-10$. The description of these results is analogous to the strip-footing problem. Therefore, we skip details. In particular, we choose $\alpha_{\max } \approx 10^{10}$ and use 36 mesh levels. The finest mesh consists of 6979 elements and 14026 nodes, and it reflects to the expected failure mechanism. Further, we compute $\psi_{h}\left(\alpha_{\max }\right) \doteq 11.55$ and $\Psi_{h}\left(\alpha_{\max }\right) \doteq 11.60$. From [9], the analytical estimate 11.65 of the limit load follows. So our guaranteed upper bound is slightly more accurate.

\section{Conclusion}

The aim of this paper was to highlight the importance of inf-sup conditions on convex cones and its consequences for limit load analysis in perfect plasticity. Although the paper is focused on this specific topic, the results were derived as much as general in order to inspire other scientists which are not familiar with plasticity.

\section{Acknowledgements}

This work was supported by The Ministry of Education, Youth and Sports of the Czech Republic from the National Programme of Sustainability (NPU II), project "IT4Innovations excellence in science - LQ1602". 

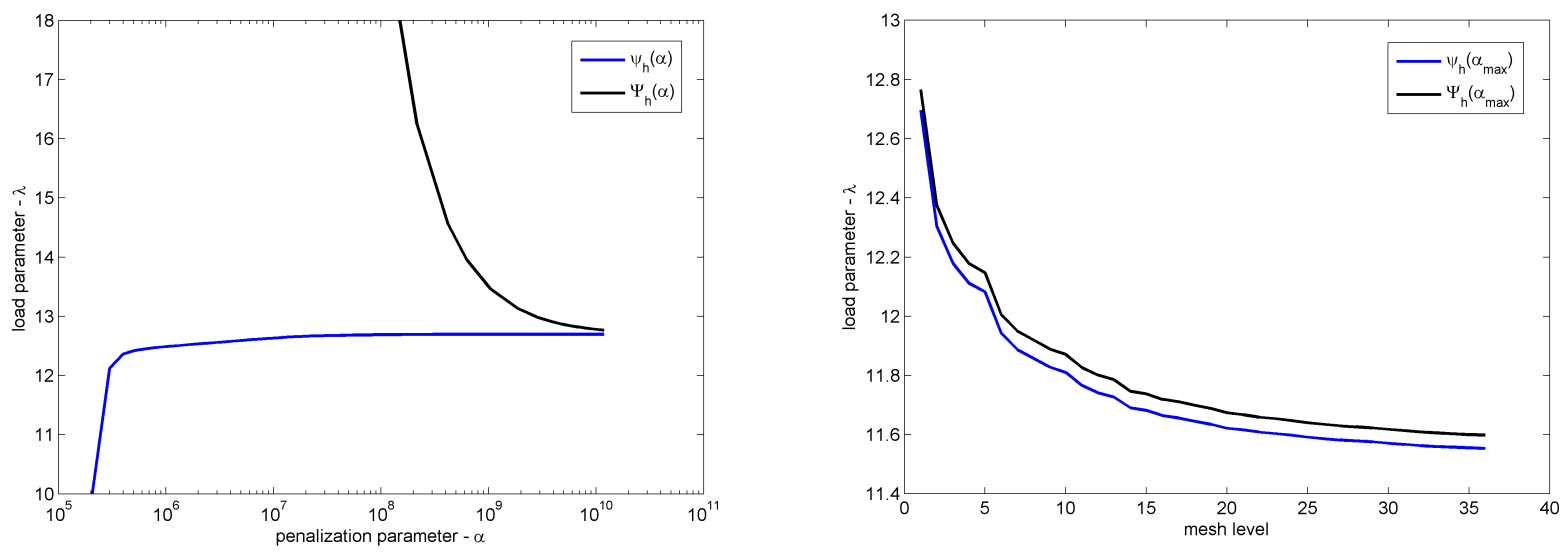

Figure 8: Comparison of $\psi_{h}$ and $\Psi_{h}$ for the slope stability problem: left $-\alpha$-convergence for the initial-coarsest mesh; right - values $\psi_{h}\left(\alpha_{\max }\right)$ and $\Psi_{h}\left(\alpha_{\max }\right)$ depending on mesh density.

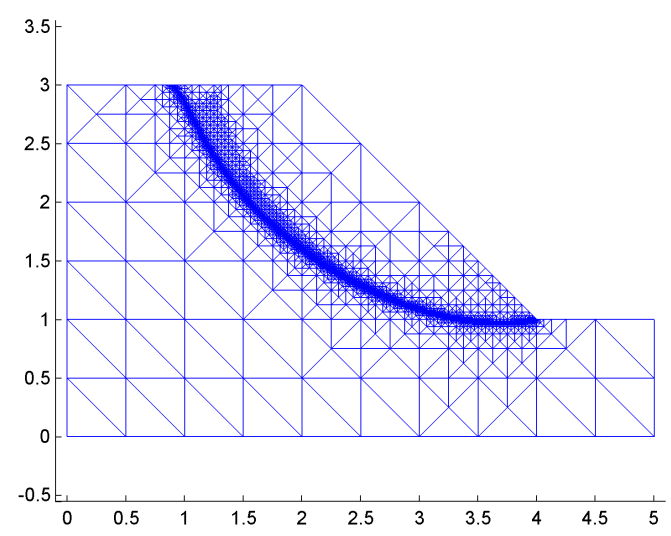

Figure 9: The finest mesh for the slope stability problem.
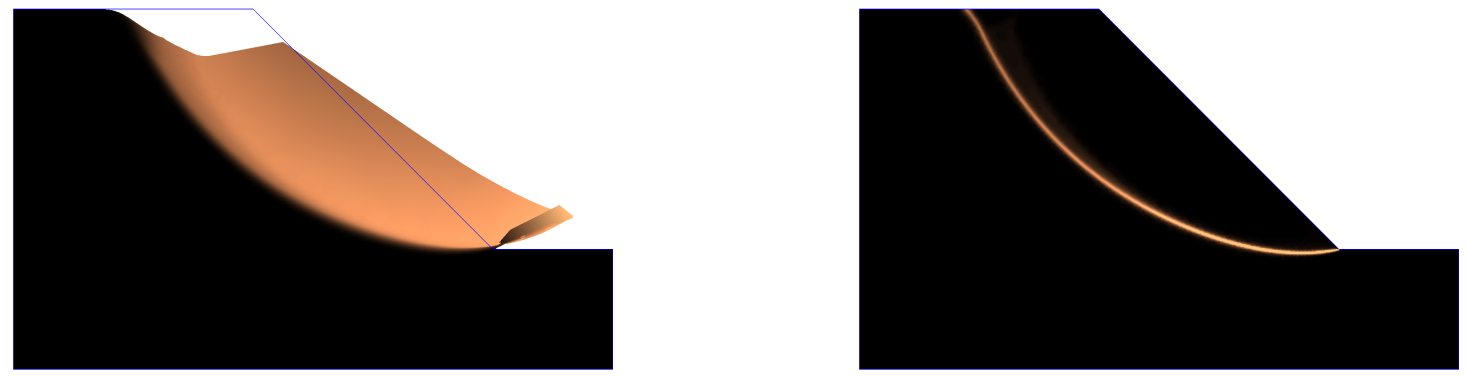

Figure 10: The field $u_{h, \alpha_{\max }}$ with enlarged deformed shapes (left) and the divergence of $u_{h, \alpha_{\max }}$ (right) for the finest mesh - the slope-stability problem. 


\section{References}

[1] I. Babuška: The finite element method with Lagrangian multipliers. Numer. Math. 20, 179-192 (1973).

[2] I. Babuška, A.K. Aziz: Survey lectures on the mathematical foundations of the finite element method. In: The Mathematical Formulations of the Finite Elements Method with Applications to the Partial Differential Equations, Academic Press, New York (1972), pp. 5-359.

[3] D. Boffi, F. Brezzi, M. Fortin: Mixed Finite Element Methods and Applications. Springer, 2013.

[4] L.A. Borges, N. Zouain, C. Costa, R. Feijóo: An adaptive approach to limit analysis. Int. J. Solids Struct. 38 (2001) 1707-1720.

[5] F. Brezzi: On the existence, uniqueness and approximation of saddle-point problems arising from Lagrange multipliers. R.I.A.R.O., Anal. Numer., R2, 129-151 (1974).

[6] A. Caboussat, R. Glowinski. Numerical solution of a variational problem arising in stress analysis: The vector case. Discret. Contin. Dyn. Syst. 27 (2010) 1447-1472.

[7] M. Cermak, J. Haslinger, T. Kozubek, S. Sysala. Discretization and numerical realization of contact problems for elastic-perfectly plastic bodies. PART II - numerical realization. ZAMM Z. Angew. Math. Mech. 95, No. 12, 1348-1371 (2015).

[8] M. Čermák, S. Sysala, J. Valdman: Fast MATLAB assembly of elastoplastic FEM matrices in 2D and 3D. ArXiv 1805.04155. Codes available in https://github.com/matlabfem/.

[9] W. Chen, X.L. Liu. Limit Analysis in Soil Mechanics. Elsevier, 1990.

[10] H. Ciria, J. Peraire, J. Bonet: Mesh adaptive computation of upper and lower bounds in limit analysis. Int. J. Numer. Methods Engng 75 (2008) 899-944.

[11] M. Costabel, M. Dauge: On the inequalities of Babuška-Aziz, Friedrichs and Horgana-Payne. Arch. Ration. Mech. Anal. 217 (2015) 873-898.

[12] E. Christiansen. Limit analysis of colapse states. In P. G. Ciarlet and J. L. Lions, editors, Handbook of Numerical Analysis, Vol IV, Part 2, North-Holland, 195-312, 1996.

[13] de Souza Neto, E. A., Perić, D., Owen, D. R. J.: Computational methods for plasticity: theory and application. Wiley, (2008).

[14] I. Ekeland, R. Temam. Analyse Convexe et Problèmes Variationnels. Dunod, Gauthier Villars, Paris, 1974.

[15] J. Haslinger, S. Repin, S. Sysala. A reliable incremental method of computing the limit load in deformation plasticity based on compliance: Continuous and discrete setting. Journal of Computational and Applied Mathematics 303 (2016) 156-170.

[16] J. Haslinger, S. Repin, S. Sysala: Guaranteed and computable bounds of the limit load for variational problems with linear growth energy functionals. Applications of Mathematics 61 (2016) 527-564.

[17] K. Krabbenhoft, A.V. Lyamin, M. Hjiaj, S.W. Sloan: A new discontinuous upper bound limit analysis formulation. Int. J. Numer. Methods Engng 63 (2005) 1069-1083. 
[18] O.A. Ladyzenskaya, V.A. Solonnikov: Some problems of vector analysis, an generalized formulations of boundary value problems for the Navier-Stokes equation. Zap. Nauchn. Semin. LOMI, 59, 81-116 (1976).

[19] A. Makrodimopoulos, C.M. Martin: Upper bound limit analysis using simplex strain elements and second-order cone programming. Int. J. Numer. Analyt. Methods Geomech. 31 (2007) 835865.

[20] J. Nečas: Les Méthodes Directes an Théorie des Équations Elliptiques. Masson et Cie, Éditeurs, Paris; Academia, Éditeurs, Prague (1967).

[21] J. Necas, I. Hlaváček: Mathematical theory of elastic and elasto-plastic bodies: an introduction (Vol. 3). Elsevier (2017).

[22] L.E. Payne: A bound for the optimal constant in an inequality of Ladyzhenskaya and Solonnikov. IMA journal of applied mathematics 72.5 (2007): 563-569.

[23] Repin, S. I. (1991). Variational formulations for discontinuous displacement fields in problems of the deformation theory of plasticity without hardening. Journal of Applied Mathematics and Mechanics, 55(6), 910-920.

[24] Repin, S. I. (1994). The solutions of problems in the mathematical theory of plasticity with discontinuities in the displacement fields. Journal of Applied Mathematics and Mechanics, 58(1), 149-160.

[25] S. Repin: A posteriori error estimates for the Stokes problem. J. Math. Sci. 109, No.5, 1950-1964 (2002).

[26] S. Repin: A Posteriori Estimates for Partial Differential Equations. Walter de Gruyter, Berlin, 2008.

[27] S. Repin: Estimates of the distance to the set of divergence free field. Journal of Mathematical Sciences 210.6 (2015): 822-834.

[28] S. Repin: Estimates of the Distance to the Set of Solenoidal Vector Fields and Applications to A Posteriori Error Control. Computational Methods in Applied Mathematics 15.4 (2015): 515-530.

[29] S. Repin: Localized forms of the LBB condition and a posteriori estimates for incompressible media problems. Mathematics and Computers in Simulation 145 (2018) 156-170.

[30] S. Repin, G. Seregin. Existence of a weak solution of the minimax problem arising in CoulombMohr plasticity, Nonlinear evolution equations, 189220, Amer. Math. Soc. Transl. (2), 164, Amer. Math. Soc., Providence, RI, (1995).

[31] S. Repin. S. Sysala, J. Haslinger: Computable majorants of the limit load in Hencky's plasticity problems. Computer and Mathematics with Applications 75 (2018) 199-217.

[32] S.W. Sloan: Geotechnical stability analysis, Géotechnique 63 (2013) 531-572.

[33] S. Sysala, J. Haslinger: Truncation and Indirect Incremental Methods in Henckys Perfect Plasticity. In: Mathematical Modelling in Solid Mechanics (pp. 265-284), 2017. Springer Singapore.

[34] S. Sysala, J. Haslinger, I. Hlaváček, and M. Cermak. Discretization and numerical realization of contact problems for elastic-perfectly plastic bodies. PART I-discretization, limit analysis. ZAMM - Z. Angew. Math. Mech. 95 (2015) 333 - 353. 
[35] R. Temam, NavierStokes equations. Theory and numerical analysis, Studies in Mathematics and its Applications, vol. 2, North-Holland, Amsterdam, 1979.

[36] R. Temam. Mathematical Problems in Plasticity. Gauthier-Villars, Paris, 1985.

[37] X. Yu and F. Tin-Loi: A simple mixed finite element for static limit analysis. Computers and Structures 84 (2006) 1906-1917. 